\title{
Compact integers and factorials
}

\author{
by \\ Vladimir Shevelev (Beer-Sheva)
}

1. Introduction. In [7], it was asked whether for every fixed positive integer $k$ there exists a positive integer $n$ so that, in the prime power factorization of $n$ !, the first $k$ primes appear with even exponents. This question was answered in the affirmative by D. Berend in [3]. In fact, Berend proved more in two aspects. First, he proved that, for arbitrary fixed $k, d \in \mathbb{N}$, there exist infinitely many numbers $n$ so that, in the prime factorization of $n$ !, the first $k$ primes appear with exponents divisible by $d$. In particular, all these exponents may be divisible by $2^{m}$ for an arbitrary $m$. Secondly, he proved that, for fixed $k \geq 1$ and $d \geq 2$, there exists a computable constant $C(k, d)$, depending only on $k, d$, such that every interval of length $C(k, d)$ of positive numbers contains a positive integer $n$ with the above property. Some natural extensions of Berend's results were obtained in [5], [6], [9], [13].

In this paper we consider factorials whose factorization

$$
n !=2^{e_{2}(n)} 3^{e_{3}(n)} \cdots p_{k}^{e_{p_{k}}(n)}
$$

is special from the point of view of one or more primes. It is easy to show that $e_{2}(n)$ is a power of 2 infinitely often. This raises the following questions.

QUESTION 1. Let $p$ be any odd prime. Is $e_{p}(n)$ a power of 2 infinitely often?

It is plausible that the answer is affirmative. However, we have not been able to prove this. Most of this paper is devoted to the following question and related issues.

Question 2. Let $p$ be any odd prime. Do there exist infinitely many positive integers $n$ for which both $e_{2}(n)$ and $e_{p}(n)$ are powers of 2 ?

2000 Mathematics Subject Classification: Primary 11A51; Secondary 11B65, 11D61, $11 \mathrm{~N} 05$.

Key words and phrases: factorization of factorials, square-free integers, diophantine equations, distribution of primes in short intervals.

The article is partly supported by a grant of the Gil'adi Foundation of the Israeli Ministry of Absorption. 
One of the main results of this paper is that the answer here is negative. We mention that this result is almost trivial for non-Fermat primes, but is trickier for Fermat primes.

Our results may be reformulated using Fermi-Dirac arithmetic ([14]). In this arithmetic, the role of primes in classical arithmetic is played by the multiplicative basis of so-called FD-primes,

$$
Q=\left\{p^{2^{k-1}}: p \in P, k \in \mathbb{N}\right\}=\{2,3,4,5,7,9,11,13,16,17, \ldots\},
$$

where $P$ denotes the set of all primes. Every positive integer $n \geq 2$ may be written uniquely in the form $n=q_{1} \cdots q_{k}$, where $q_{1}, \ldots, q_{k}$ are distinct FD-primes, and we shall write $Q_{n}=\left\{q_{1}, \ldots, q_{k}\right\}$ in this case. We put $Q_{1}=\emptyset$.

Definition 1. A positive integer $n$ is compact if all elements of $Q_{n}$ are relatively prime.

Denote the set of all compact numbers by $C$. It is convenient to suppose that $1 \in C$. In Theorem 1 we find the density of the set $C$, along with an error term for its counting function.

It is easy to see that the set

$$
C^{!}=\{n \in \mathbb{N}: n ! \in C\}
$$

is finite. In fact, if $n$ is sufficiently large, then the interval $(n / 4, n / 3)$ contains a prime $p$, and it is easy to verify that $p^{3} \| n$ !, i.e. $e_{p}(n)=3$. Below we obtain the following result: $C^{!}=\{1,2,3,6,7,10,11\}$.

Definition 2. Let $p$ be a fixed prime. A positive integer $n$ divisible by $p$ is $p$-compact if the set $Q_{n}$ contains a single power of $p$.

Denote the set of $p$-compact numbers by $C_{p}$ and put

$$
C_{p}^{!}=\left\{n \in \mathbb{N}: n ! \in C_{p}\right\} .
$$

Our answer to Question 2 may be rephrased as the statement that, for each prime $p \geq 3$ the set $C_{2}^{!} \cap C_{p}^{!}$is finite. Moreover, we obtain an explicit formula for $\left|C_{2}^{!} \cap C_{p}^{!}\right|$. In our approach, we are led to consider various exponential diophantine equations. Our formula for the size of $C_{2}^{!} \cap C_{p}^{!}$also allows us to compute the lower and upper densities of those sets of primes for which this size assumes any specific value. Moreover, we obtain an estimate for the least prime for which this set is of some given size. Our estimates depend on the up-to-date results regarding the number of primes in short intervals.

In Section 2 we present the main results. Sections 3-8 are devoted to the proofs. In Section 9 we provide some numerical results. Finally, in Section 10 we pose several questions for further research. 
2. The main results. Let

$$
c(x)=\sum_{i \leq x, i \in C} 1, \quad c_{p}(x)=\sum_{i \leq x, i \in C_{p}} 1 .
$$

THEOREM 1.

(i) For every $x \geq 1$,

$$
c(x)=\lambda x+R(x)
$$

where

$$
\begin{aligned}
& \lambda=\frac{6}{\pi^{2}} \prod_{p \in P}\left(1+\frac{1}{p+1} \sum_{i=1}^{\infty} p^{-\left(2^{i}-1\right)}\right)=0.872497 \ldots, \\
& |R(x)| \leq \begin{cases}k_{1}\left(\log x+k_{2}\right) \sqrt{x} & \text { if } x \leq 4 \cdot 10^{19}, \\
k_{1}\left(\log x+k_{2}+e^{\left.k_{3} \frac{\sqrt{\log x}}{\log \log \sqrt{x}} \log \frac{x}{4 \cdot 10^{19}}\right) \sqrt{x}}\right. & \text { if } x>4 \cdot 10^{19}\end{cases}
\end{aligned}
$$

with $k_{1}=28.841303 \ldots, k_{2}=0.152970 \ldots, k_{3}=5.263054 \ldots$.

(ii) For any fixed prime $p$,

$$
c_{p}(x)=\beta_{p} x+O(\log \log x)
$$

with the constant in $O(.$.$) equal to 1 / \log 2$, where

$$
\beta_{p}=\frac{p-1}{p} \sum_{i=0}^{\infty} p^{-2^{i}}
$$

THEOREM 2.

$$
\left|C_{2}^{!} \cap C_{q}^{!}\right|= \begin{cases}7, & q=3, q=5, \\ 6, & q=7, \\ 5, & q=\left(2^{4 k+1}+3\right) / 5, \quad k \geq 2, \\ 4, & q=2^{2^{k-1}}+1, \quad k \geq 3, \\ 3, & q=2^{k}+3, k \geq 3, \\ 2\left(1+\left\lfloor\log _{2} \frac{q-5}{2^{k}-q}\right\rfloor\right), & 2^{k-1}+3<q \leq 2^{k}-1, k \geq 4, \\ & q \neq\left(2^{k+2}+3\right) / 5 .\end{cases}
$$

Theorem 3. For a fixed $t \in \mathbb{N}$,

$$
\left|\left\{q \in P, q \leq 2^{m}:\left|C_{2}^{!} \cap C_{q}^{!}\right|=2 t\right\}\right| \sim \frac{2^{t}}{\left(2^{t-1}+1\right)\left(2^{t}+1\right)} \pi\left(2^{m}\right) ;
$$

moreover,

$$
\begin{aligned}
& \limsup _{n \rightarrow \infty} \frac{\left|\left\{q \in P, q \leq n:\left|C_{2}^{!} \cap C_{q}^{!}\right|=2 t\right\}\right|}{\pi(n)}=\frac{1}{2^{t-1}+1}, \\
& \liminf _{n \rightarrow \infty} \frac{\left|\left\{q \in P, q \leq n:\left|C_{2}^{!} \cap C_{q}^{!}\right|=2 t\right\}\right|}{\pi(n)}=\frac{1}{2^{t}+1} .
\end{aligned}
$$


THEOREM 4. For sufficiently large $k$, as q varies over $\left(2^{k-1}+3,2^{k}-1\right)$ :

(i) $\left|C_{2}^{!} \cap C_{q}^{!}\right|$assumes all even values in the interval $[2,0.95 k-2]$;

(ii) the number of primes $q \in\left(2^{k-1}+3,2^{k}-1\right)$ for which $\left|C_{2}^{!} \cap C_{q}^{!}\right|=2 t$ with $t \in[1,0.475 k-1]$ is not less than

$$
\frac{0.09}{\log 2} \frac{2^{0.525(k-1)}}{k} .
$$

REMARK 1. The proof depends on the estimates regarding the number of primes in short intervals [2]. Improvements in these estimates will imply corresponding improvements in Theorem 4, namely that $\left|C_{2}^{!} \cap C_{q}^{!}\right|$assumes all even values in a larger interval.

For a given $t \in \mathbb{N}$ consider the function

$$
q(t)=\min \left\{q \in P:\left|C_{2}^{!} \cap C_{q}^{!}\right|=2 t\right\} .
$$

THEOREM 5. For sufficiently large $t$,

$$
2^{t}-1 \leq q(t) \leq 2^{\lceil 40 t / 19\rceil} .
$$

REMARK 2. Similarly to Remark 1 improvements in the above mentioned estimates will imply corresponding improvements of the upper bound for $q(t)$.

3. Proof of Theorem 1. Let $r$ be a fixed square-free number. Consider the auxiliary function $\beta_{r}(x)$, defined as the number of positive integers not exceeding $x$, not divisible by the square of any prime, except perhaps the prime divisors of $r$.

LeMMA 1. For every $x \geq 1$,

$$
\beta_{r}(x)=\frac{6}{\pi^{2}} \prod_{p \mid r}\left(1-\frac{1}{p^{2}}\right)^{-1} x+\varrho(x),
$$

where $|\varrho(x)| \leq 3.5 \sqrt{x}$.

Proof. By inclusion-exclusion

$$
\begin{aligned}
\beta_{r}(x) & =\lfloor x\rfloor-\sum_{\substack{p \leq x \\
p \nmid r}}\left\lfloor\frac{x}{p^{2}}\right\rfloor+\sum_{\substack{p<q \leq x \\
p, q \nmid r}}\left\lfloor\frac{x}{p^{2} q^{2}}\right\rfloor-\cdots \\
& =\sum_{i \leq x} \sum_{\substack{d^{2} \mid i \\
(d, r)=1}} \mu(d)=\sum_{\substack{d \leq \sqrt{x} \\
(d, r)=1}} \mu(d) \sum_{\substack{i \leq x \\
d^{2} \mid i}} 1 \\
& =\sum_{\substack{d \leq \sqrt{x} \\
(d, r)=1}} \mu(d)\left\lfloor\frac{x}{d^{2}}\right\rfloor=x \sum_{\substack{d \leq \sqrt{x} \\
(d, r)=1}} \frac{\mu(d)}{d^{2}}+R(x),
\end{aligned}
$$


where

$$
|R(x)| \leq \frac{1}{2} \sum_{d \leq \sqrt{x}}|\mu(d)|+o(\sqrt{x})=\frac{1}{2} \beta_{1}(\sqrt{x})+o(\sqrt{x}) .
$$

The coefficient $1 / 2$ in (2) follows from the well known estimate $\left|\sum_{n \leq x} \mu(n)\right|$ $=o(x)($ see $[17])$. Now

$$
\begin{aligned}
\sum_{\substack{d=1 \\
(d, r)=1}}^{\infty} \frac{\mu(d)}{d^{2}} & =\prod_{\substack{p \in P \\
p \nmid r}}\left(1-\frac{1}{p^{2}}\right)=\prod_{p \in P}\left(1-\frac{1}{p^{2}}\right) \prod_{p \mid r}\left(1-\frac{1}{p^{2}}\right)^{-1} \\
& =\frac{6}{\pi^{2}} \prod_{p \mid r}\left(1-\frac{1}{p^{2}}\right)^{-1}
\end{aligned}
$$

and therefore

$$
\sum_{\substack{d \leq \sqrt{x} \\(d, r)=1}} \frac{\mu(d)}{d^{2}}=\frac{6}{\pi^{2}} \prod_{p \mid r}\left(1-\frac{1}{p^{2}}\right)^{-1}-\sum_{\substack{d>\sqrt{x} \\(d, r)=1}} \frac{\mu(d)}{d^{2}} .
$$

We estimate the second term on the right-hand side trivially:

$$
\left|\sum_{\substack{d>\sqrt{x} \\(d, r)=1}} \frac{\mu(d)}{d^{2}}\right| \leq \sum_{d \geq\lfloor\sqrt{x}\rfloor+1} \frac{1}{d^{2}} \leq \int_{\lfloor\sqrt{x}\rfloor}^{\infty} \frac{d t}{t^{2}}=\frac{1}{\lfloor\sqrt{x}\rfloor}
$$

It is easy to see that $\sup _{x \geq 1} \sqrt{x} /\lfloor\sqrt{x}\rfloor=\sqrt{3.99 \ldots / 1}=2$. So, by $(4)$,

$$
x\left|\sum_{\substack{d>\sqrt{x} \\(d, r)=1}} \frac{\mu(d)}{d^{2}}\right| \leq 2 \sqrt{x} .
$$

Taking into account that in $(2) o(\sqrt{x}) \leq \sqrt{x}$ for $x \geq 1$, by (1)-(5), for every $x$ we have

$$
\beta_{r}(x)=\frac{6}{\pi^{2}} \prod_{p \mid r}\left(1-\frac{1}{p^{2}}\right)^{-1} x+\varrho(x)
$$

where $|\varrho(x)| \leq \sqrt{x}(1 / 2+1+2)=3.5 \sqrt{x}$.

REMARK 3. It follows from the proof of Lemma 1 that for $x$ large enough we have $\varrho(x) \leq\left(1+3 / \pi^{2}+\varepsilon\right) \sqrt{x}$.

For a fixed square-free number $r$, denote by $B_{r}$ the set of square-free numbers $n$ for which $(n, r)=1$, and put

$$
b_{r}(x)=\left|B_{r} \cap\{1,2, \ldots, x\}\right| .
$$

In particular, $B=B_{1}$ is the set of all square-free numbers. 
Lemma 2.

$$
b_{r}(x)=\frac{6 r}{\pi^{2}} \prod_{p \mid r}(p+1)^{-1} x+R_{r}(x),
$$

where for every $x \geq 1$ and every $r \in B$,

$$
\left|R_{r}(x)\right| \leq \begin{cases}57.682607 \ldots \sqrt{x} & \text { if } r \leq N \\ 57.682607 \ldots e^{7.443083 \ldots \frac{\sqrt{\log r}}{\log \log r}} \sqrt{x} & \text { if } r \geq N+1,\end{cases}
$$

where $N=6469693229$.

Proof. Consider the function $\lambda: P \rightarrow\{1,2\}$ defined by

$$
\lambda(p)= \begin{cases}1, & p \mid r \\ 2, & p \nmid r .\end{cases}
$$

By inclusion-exclusion

$$
b_{r}(x)=\lfloor x\rfloor-\sum_{p \leq x}\left\lfloor\frac{x}{p^{\lambda(p)}}\right\rfloor+\sum_{p<q \leq x}\left\lfloor\frac{x}{p^{\lambda(p)} q^{\lambda(p)}}\right\rfloor-\cdots,
$$

where all sums are over primes only. It follows from (6) that

$$
\begin{aligned}
b_{r}(x)= & \left(\lfloor x\rfloor-\sum_{\substack{p \leq x \\
p \nmid r}}\left\lfloor\frac{x}{p^{2}}\right\rfloor+\sum_{\substack{p<q \leq x \\
p, q \nmid r}}\left\lfloor\frac{x^{2}}{p^{2} q^{2}}\right\rfloor-\cdots\right) \\
& -\sum_{p_{1} \mid r}\left(\left\lfloor\frac{x}{p_{1}}\right\rfloor-\sum_{\substack{p \leq x \\
p \nmid r}}\left\lfloor\frac{x}{p_{1}} / p^{2}\right\rfloor+\sum_{\substack{p<q \leq x \\
p, q \nmid r}}\left\lfloor\frac{x}{p_{1}} / p^{2} q^{2}\right\rfloor-\cdots\right) \\
& +\sum_{p_{1}<p_{2}: p_{1} p_{2} \mid r}\left(\left\lfloor\frac{x}{p_{1} p_{2}}\right\rfloor-\sum_{\substack{p \leq x \\
p \nmid r}}\left\lfloor\frac{x}{p_{1} p_{2}} / p^{2}\right\rfloor+\sum_{\substack{p<q \leq x \\
p, q \nmid r}}\left\lfloor\frac{x}{p_{1} p_{2}} / p^{2} q^{2}\right\rfloor-\cdots\right) \\
& -\cdots \\
= & \sum_{d \mid r} \mu(d) \beta_{r}\left(\frac{x}{d}\right) .
\end{aligned}
$$

Therefore, by Lemma 1 ,

$$
\begin{aligned}
b_{r}(x) & =\frac{6}{\pi^{2}} \prod_{p \mid r}\left(1-\frac{1}{p^{2}}\right)^{-1} x \sum_{d \mid r} \frac{\mu(d)}{d}+R_{r}(x) \\
& =\frac{6}{\pi^{2}} \prod_{p \mid r}\left(1-\frac{1}{p^{2}}\right)^{-1} \prod_{p \mid r}\left(1-\frac{1}{p}\right) x+R_{r}(x) \\
& =\frac{6}{\pi^{2}} \prod_{p \mid r} \frac{p}{p+1} x+R_{r}(x)=\frac{6 r}{\pi^{2}} \prod_{p \mid r}(p+1)^{-1} x+R_{r}(x),
\end{aligned}
$$


where

$$
\left|R_{r}(x)\right| \leq 3.5 \sqrt{x} \sum_{d \mid r} \frac{1}{\sqrt{d}}=3.5 \prod_{p \mid r}\left(1+\frac{1}{\sqrt{p}}\right) \sqrt{x}
$$

Let $w(r)$ denote the number of prime divisors of $r$. If $w(r) \leq 9$, then

$$
\prod_{p \mid r}\left(1+\frac{1}{\sqrt{p}}\right) \leq \prod_{2 \leq p \leq 23}\left(1+\frac{1}{\sqrt{p}}\right)=16.480745 \ldots
$$

If $w(r) \geq 10$, then $r \geq \prod_{2 \leq p \leq 29} p=6469693230$. Put $m=p_{w(r)} \geq 29$.

For $m \geq 29$, as is well known (cf. [16]), $\prod_{2 \leq p \leq m} p>2^{m}$. Therefore, if $w(r) \geq 10$, then $2^{m}<\prod_{p \mid r} p=r$, i.e. $m<\log _{2} r$. Taking into account that the $n$th prime satisfies $p_{n} \geq n \log n$, and $\pi(m) \leq 1.6 m / \log m$ for every $m \geq 2$ (cf. [16]), we have

$$
\begin{gathered}
\log \prod_{p \mid r}\left(1+\frac{1}{\sqrt{p}}\right)-\log \prod_{p \leq 23}\left(1+\frac{1}{\sqrt{p}}\right) \\
\leq \log \prod_{p \leq m}\left(1+\frac{1}{\sqrt{p}}\right)-\log \prod_{p \leq 23}\left(1+\frac{1}{\sqrt{p}}\right) \leq \sum_{29 \leq p \leq m} \frac{1}{\sqrt{p}} \\
=\sum_{n: 29 \leq p_{n} \leq m} \frac{1}{\sqrt{p}} \leq \sum_{n=10}^{\pi(m)} \frac{1}{\sqrt{n \log n}} \\
\leq \int_{9}^{1.6 m / \log m} \frac{d t}{\sqrt{t \log t}}=\int_{9}^{1.6 m / \log m} \frac{t^{1 / 4}}{\sqrt{\log t}} \frac{d t}{t^{3 / 4}}
\end{gathered}
$$

Notice that for $t>e^{2}$ the function $t^{1 / 4} / \sqrt{\log t}$ increases. Therefore, since for $m \geq 9$ we have $\log \log m<\frac{1}{3} \log (1.6 m)$,

$$
\begin{aligned}
\log \prod_{p \mid r} & \left(1+\frac{1}{\sqrt{p}}\right)-\log 16.480745 \ldots \\
& \leq\left(1.6 \frac{m}{\log m}\right)^{1 / 4}(\log 1.6+\log m-\log \log m)^{-1 / 2} \int_{9}^{1.6 m / \log m} \frac{d t}{t^{3 / 4}} \\
& \leq 4\left(1.6 \frac{m}{\log m}\right)^{1 / 2}\left(\frac{2}{3} \log m\right)^{-1 / 2} \\
& =4 \sqrt{24} \frac{m^{1 / 2}}{\log m}<4 \sqrt{\frac{2.4}{\log 2}} \frac{\sqrt{\log r}}{\log \log r} .
\end{aligned}
$$


Thus, for $N=6469693229$ and every $r \in B$,

$$
\prod_{p \mid r}\left(1+\frac{1}{\sqrt{p}}\right) \leq \begin{cases}16.480745 \ldots & \text { if } r \leq N \\ 16.480745 \ldots e^{7.443083 \ldots \frac{\sqrt{\log r}}{\log \log r}} & \text { if } r \geq N+1 .\end{cases}
$$

By (7) and (8) we obtain the conclusion of the lemma.

Now we can complete the proof of Theorem 1(i). Let $a \geq 1$ be a compact number. Denote by $r(a)$ the product of all prime divisors of $a$; set $r(1)=1$. Consider, further, the subset $C^{(a)}$ of the compact numbers of the form $a^{2} s$, where $a \in C$ and $s \in B_{r(a)}$. It is evident that, if $a_{1} \neq a_{2}$, then $C^{\left(a_{1}\right)} \cap C^{\left(a_{2}\right)}$ $=\emptyset$, and therefore $C=\bigcup_{a \in C} C^{a}$, where the union is disjoint. Consequently, by Lemma 2 ,

$$
\begin{aligned}
c(x) & =b_{1}(x)+\sum_{\substack{2 \leq a \leq \sqrt{x}, a \in C\\
}} b_{r}(a)\left(\frac{x}{a^{2}}\right) \\
& =\frac{6}{\pi^{2}}\left(1+\sum_{\substack{2 \leq a \leq \sqrt{x} \\
a \in C}} \prod_{p \mid r(a)}\left(1-\frac{1}{p+1}\right) \frac{1}{a^{2}}\right) x+R^{*}(x),
\end{aligned}
$$

where

$$
\begin{aligned}
\left|R^{*}(x)\right| & \leq 3.5 \sqrt{x}+\sum_{\substack{2 \leq a \leq \sqrt{x} \\
a \in C}}\left|R_{r(a)}\left(\frac{x}{a^{2}}\right)\right| \\
& \leq 3.5 \sqrt{x}+\sum_{\substack{2 \leq a \leq \sqrt{x}: r(a)=N \\
a \in C}}\left|R_{r(a)}\left(\frac{x}{a^{2}}\right)\right|+\sum_{\substack{a \leq \sqrt{x}: r(a) \geq N+1 \\
a \in C}}\left|R_{r(a)}\left(\frac{x}{a^{2}}\right)\right|
\end{aligned}
$$

with $N=6469693229$. Therefore, if $x \leq N^{2}$, then

$$
\left|R^{*}(x)\right| \leq\left(3.5+k_{1} \log x\right) \sqrt{x},
$$

where $k_{1}=28.841303 \ldots$ If $x>N^{2}$, then

$$
\begin{aligned}
\left|R^{*}(x)\right| \leq & \left(3.5+k_{1} \log x\right) \sqrt{x} \\
& +k_{1} \sqrt{x} \sum_{\substack{a \leq \sqrt{x}: r(a) \geq N+1 \\
a \in C}} \frac{1}{a} e^{7.443083 \ldots \frac{\sqrt{\log r(a)}}{\log \log r(a)}},
\end{aligned}
$$

where the last sum does not exceed

$$
\sum_{N+1 \leq a \leq \sqrt{x}: r(a) \geq N+1} \frac{1}{a} e^{7.443083 \ldots \frac{\sqrt{\log a}}{\log \log a}} \leq e^{k_{3} \frac{\sqrt{\log x}}{\log \log \sqrt{x}}} \log \frac{\sqrt{x}}{N}
$$

with $k_{3}=7.443083 / \sqrt{2}=5.263054 \ldots$.

Moreover, if we replace in (10) the sum $\sum_{a \leq \sqrt{x}, a \in C}$ by $\sum_{a \in C}$, then the error does not exceed (see (4)) 


$$
\frac{6 x}{\pi^{2}} \sum_{n>\sqrt{x}} \frac{1}{n^{2}} \leq \frac{6}{\pi^{2}}\left(\sup _{x \geq 4} \frac{\sqrt{x}}{\lfloor\sqrt{x}\rfloor}\right) \sqrt{x}=\frac{6}{\pi^{2}} \frac{\sqrt{8.999 \ldots}}{2} \sqrt{x}=0.911890 \ldots \sqrt{x} .
$$

Thus,

$$
c(x)=\frac{6 x}{\pi^{2}} \sum_{a \in C} \prod_{p \mid r(a)}\left(1-\frac{1}{p+1}\right) \frac{1}{a^{2}}+R(x),
$$

where $R(x)=R^{*}(x)+0.911890 \ldots \sqrt{x}$. Hence

$$
|R(x)| \leq \begin{cases}k_{1}\left(\log x+k_{2}\right) \sqrt{x} & \text { if } x \leq N^{2}, \\ k_{1}\left(\log x+k_{2}+e^{\left.k_{3} \frac{\sqrt{\log x}}{\log \log \sqrt{x}} \log \frac{x}{N^{2}}\right) \sqrt{x}}\right. & \text { if } x>N^{2},\end{cases}
$$

with $N=6469693229, k_{1}=28.841303 \ldots, k_{2}=(3.5+0.911890 \ldots) / k_{1}=$ $0.152970 \ldots, k_{3}=5.263054 \ldots$

It remains to evaluate the sum (11). For a fixed $l \in B$, denote by $C(l)$ the set of all compact numbers $a$ with $r(a)=l$. By (11),

$$
c(x)=\frac{6 x}{\pi^{2}} \sum_{l \in B} \prod_{p \mid l}\left(1-\frac{1}{p+1}\right) \sum_{a \in C(l)} \frac{1}{a^{2}}+R(x) .
$$

Consider the function $A: \mathbb{N} \rightarrow \mathbb{R}$ given by

$$
A(l)= \begin{cases}\sum_{a \in C(l)} 1 / a^{2}, & l \in B, \\ 0, & l \notin B .\end{cases}
$$

It is evident that, if $l_{1}, l_{2} \in B$ and $\left(l_{1}, l_{2}\right)=1$, then

$$
A\left(l_{1} l_{2}\right)=\sum_{a \in C\left(l_{1} l_{2}\right)} \frac{1}{a^{2}}=\sum_{a \in C\left(l_{1}\right)} \frac{1}{a^{2}} \sum_{a \in C\left(l_{2}\right)} \frac{1}{a^{2}}=A\left(l_{1}\right) A\left(l_{2}\right) .
$$

It follows that $A(l)$ is a multiplicative function. Hence the function $f$ defined by

$$
f(l)=\prod_{p \mid l}\left(1-\frac{1}{p+1}\right) A(l)
$$

is also multiplicative. Consequently ([4, p. 103]),

$$
\sum_{n=1}^{\infty} f(n)=\prod_{p \in P}\left(1+f(p)+f\left(p^{2}\right)+\cdots\right) .
$$


Since $f\left(p^{k}\right)=0$ for $k \geq 2$, by (13) we have

$$
\begin{aligned}
c(x) & =\frac{6 x}{\pi^{2}} \sum_{l=1}^{\infty} f(l)+R(x)=\frac{6 x}{\pi^{2}} \prod_{p \in P}(1+f(p))+R(x) \\
& =\frac{6 x}{\pi^{2}} \prod_{p \in P}\left(1+\left(1-\frac{1}{p+1}\right)\left(\frac{1}{p^{2}}+\frac{1}{p^{4}}+\frac{1}{p^{8}}+\cdots\right)\right)+R(x) \\
& =\frac{6}{\pi^{2}} \prod_{p \in P}\left(1+\frac{1}{p+1}\left(\frac{1}{p}+\frac{1}{p^{3}}+\frac{1}{p^{7}}+\frac{1}{p^{15}}+\cdots\right)\right) x+R(x) .
\end{aligned}
$$

Employing the estimate of $R(x)$ given by (12) (with $4 \cdot 10^{19}<N^{2}$ ), we obtain assertion (i) of the theorem.

To prove (ii), note that it is evident that

$$
\begin{aligned}
c_{p}(n) & =\left(\left\lfloor\frac{n}{p}\right\rfloor-\left\lfloor\frac{n}{p^{2}}\right\rfloor\right)+\left(\left\lfloor\frac{n}{p^{2}}\right\rfloor-\left\lfloor\frac{n}{p^{3}}\right\rfloor\right)+\left(\left\lfloor\frac{n}{p^{4}}\right\rfloor-\left\lfloor\frac{n}{p^{5}}\right\rfloor\right)+\cdots \\
& =\left(\left\lfloor\frac{n}{p}\right\rfloor-\left\lfloor\frac{n}{p^{3}}\right\rfloor\right)+\left(\left\lfloor\frac{n}{p^{4}}\right\rfloor-\left\lfloor\frac{n}{p^{5}}\right\rfloor\right)+\cdots
\end{aligned}
$$

Let

$$
p^{2^{d-1}} \leq n<p^{2^{d}}, \quad d \in \mathbb{N} .
$$

If $d \geq 2$ we have exactly $d-1$ nonzero terms in brackets. Since

$$
d-1 \leq \frac{\log \log n-\log \log p}{\log 2}
$$

we have

$$
c_{p}(n)=\frac{n}{p}-\frac{n}{p^{2}}+\frac{n}{p^{2}}-\frac{n}{p^{3}}+\cdots+\frac{n}{p^{2^{d-1}}}-\frac{n}{p^{2^{d}+1}}+O(\log \log n),
$$

where the constant in $O(\ldots)$ equals $1 / \log 2$. Hence

$$
\begin{aligned}
c_{p}(n) & =n \frac{p-1}{p}\left(\frac{1}{p}+\frac{1}{p^{2}}+\frac{1}{p^{4}}+\cdots+\frac{1}{p^{2^{d-1}}}\right)+O(\log \log n) \\
& =n \frac{p-1}{p} \sum_{i=0}^{\infty} p^{-2^{i}}-n \frac{p-1}{p} \sum_{i=d}^{\infty} p^{-2^{i}}+O(\log \log n),
\end{aligned}
$$

and it suffices to notice that

$$
n \frac{p-1}{p} \sum_{i=d}^{\infty} p^{-2^{i}}<\frac{p-1}{p}\left(1+\frac{1}{p}+\frac{1}{p^{2}}+\cdots\right)=1 .
$$

This completes the proof.

REMARK 4. In Theorem 1(i), we can reduce $k_{3}$ to $\sqrt{2}+\varepsilon$ (at the expense of enlarging $k_{1}$ ). In fact, for small $\delta>0$ and large enough $m=m(\delta)=$ $e^{2.25 / \delta^{2}}$, according to B. Rosser [12], $\prod_{2 \leq p \leq m} p>e^{\left(1-4 \delta^{2} / 3\right) m}$, and we can 
replace (9) by

$$
\log \prod_{p \leq m}\left(1+\frac{1}{\sqrt{p}}\right) \leq K(\delta)+\int_{e^{1 / 2 \delta}}^{m /(\log m-4)} \frac{t^{\delta}}{\sqrt{\log t}} \frac{d t}{t^{1 / 2+\delta}}, \quad m>m(\delta)
$$

$(m(\delta)$ was chosen with something to spare to satisfy the technical inequality $\left.(1-3 \delta) \log m<(1-2 \delta)((\log m-4)(\log (\log m-4)))^{1 / 2}\right)$. Then we obtain for $x \geq 3$, instead of $(12)$,

$$
R(x) \leq K_{\varepsilon} \sqrt{x} e^{(\sqrt{2}+\varepsilon) \frac{(\log x)^{1 / 2}}{\log \log x}} \log x .
$$

It can be shown that we can take $K_{\varepsilon}=e^{e^{21 \varepsilon^{-2}}}$.

\section{Proof of Theorem 2}

4.1. Auxiliary propositions. Denote by $\sigma_{g}(n)$ the sum of digits in the base $g$ representation of $n \in \mathbb{N}$.

LEMMA $3([11])$.

$$
\sigma_{g}(n)=n-(g-1) \sum_{t \geq 1}\left\lfloor\frac{n}{g t}\right\rfloor, \quad n \in \mathbb{N} .
$$

By Lemma 3 we may express $n$ in terms of $\sigma_{p}(n)$ and the exponent $e_{p}(n)$ for which $p^{e_{p}(n)} \| n !$ :

$$
n=(p-1) e_{p}(n)+\sigma_{p}(n)
$$

In [11], the estimate $\sigma_{g}(n) \leq(g-1) \log _{g}(g n)$ is proved. The following lemma improves this bound, which will be useful later.

LEMMA 4.

$$
\sigma_{g}(n) \leq(g-1) \log _{g}(n+1) .
$$

Proof. Let $\sigma_{g}(n) \equiv r(\bmod g-1)$, where $0 \leq r \leq g-2$. Given any $k$, the smallest $n$ for which $\sigma_{g}(n)=(g-1) k+r$ is

$$
\begin{aligned}
n & =r g^{k}+(g-1)\left(g^{k-1}+g^{k-2}+\cdots+1\right)=(r+1) g^{k}-1 \\
& =(r+1) g^{\left(\sigma_{g}(n)-r\right) /(g-1)}-1 .
\end{aligned}
$$

Hence for every $n$ we have

$$
\sigma_{g}(n) \leq r+(g-1) \log _{g}(n+1)-(g-1) \log _{g}(r+1)
$$

Now for $r=0$ the lemma follows directly, while for $r \geq 1$ we have $r \leq$ $(g-1) \log (r+1) / \log g$, and so $1 \leq r \leq g-2$, since $x / \log (x+1)$ increases at least for $x \geq 1$.

From Lemmas 3 and 4 we obtain the following estimate. $<0$.

Corollary 1. $e_{p}(n)=n /(p-1)+R_{p}(n)$, where $-\log _{p}(n+1) \leq R_{p}(n)$ 
Proposition 1. For $n \geq 6$ we have $n \in C_{2}^{!}$if and only if there exists an $\alpha \geq 2$ such that $n=2^{\alpha}+2$ or $n=2^{\alpha}+3$.

Proof. If $n$ is of the required form, then

$$
e_{2}(n)=2^{\alpha-1}+1+2^{\alpha-2}+\cdots+1=2^{\alpha}, \quad \alpha \geq 2,
$$

and thus $n \in C_{2}^{!}$.

Now assume that $n \in C_{2}^{!}, n \geq 6$. By (15) there exists some $\alpha \in \mathbb{N}$ so that $e_{2}(n)=2^{\alpha}$ and

$$
n=e_{2}(n)+\sigma_{2}(n)=2^{\alpha}+\sigma_{2}(n)
$$

Further, by Lemma 4,

$$
\sigma_{2}(n) \leq\left\lfloor\log _{2}(n+1)\right\rfloor<n / 2, \quad n \geq 6 .
$$

Therefore, by (16) we have $2^{\alpha}<n<2^{\alpha}+n / 2$ and consequently $2^{\alpha}<n<$ $2^{\alpha+1}$, so that $\alpha=\left\lfloor\log _{2} n\right\rfloor$. Taking this into account, we apply $\sigma_{2}$ to both sides of (16):

$$
\sigma_{2}(n)=\sigma_{2}\left(2^{\left\lfloor\log _{2} n\right\rfloor}+\sigma_{2}(n)\right) .
$$

Since $\sigma_{2}(n) \leq 1+\left\lfloor\log _{2}(n)\right\rfloor<2^{\left\lfloor\log _{2}(n)\right\rfloor}$, by (17) we have

$$
\sigma_{2}(n)=1+\sigma_{2}\left(\sigma_{2}(n)\right), \quad n \geq 6
$$

Now by (18), and Lemma 3 for $g=2$,

$$
\sigma_{2}(n)=1+\sigma_{2}(n)-\sum_{t \geq 1}\left\lfloor\frac{\sigma_{2}(n)}{2^{t}}\right\rfloor
$$

and therefore $\sum_{t>1}\left\lfloor\sigma_{2}(n) / 2^{t}\right\rfloor=1$. It follows that $\sigma_{2}(n)=2$ or 3 , and (16) implies that $n=2^{\alpha}+2$ or $n=2^{\alpha}+3$.

Proposition 2. If $q$ is an odd prime, then $\left|C_{2}^{!} \cap C_{q}^{!}\right| \geq 2$.

Proof. If $q=3$, then $3,6 \in C_{2}^{!} \cap C_{3}^{!}$. Let $q \geq 5$. Put $k=\left\lceil\log _{2}(q+1)\right\rceil$. Then $k \geq 3$ and $2^{k-1}+1 \leq q \leq 2^{k}-1$. If $q=2^{k-1}+1$, then by Proposition 1 we have $q+1, q+2 \in C_{2}^{!} \cap C_{q}^{!}$. If $q \geq 2^{k-1}+3$, then $2 q \geq 2^{k}+6$ and

$$
q+3 \leq 2^{k}+2<2^{k}+3<2 q .
$$

Therefore, $\left\lfloor\left(2^{k}+2\right) / q\right\rfloor=\left\lfloor\left(2^{k}+3\right) / q\right\rfloor=1$, i.e. $2^{k}+2,2^{k}+3 \in C_{2}^{!} \cap C_{q}^{!}$.

Later we shall see that the minimal $q$ for which $\left|C_{2}^{!} \cap C_{q}^{!}\right|=2$ is $q=37$.

Proposition 3. If $p<q$ are primes so that $(q-1) /(p-1)$ is not a power of 2 , then the set $C_{p}^{!} \cap C_{q}^{!}$is finite. Moreover, if $n \in C_{p}^{!} \cap C_{q}^{!}$, then

$$
n<2\left(1+\frac{\log 2}{2 \log q-1}\right) q^{2}-1
$$


Proof. We have $(p-1) / \log p<(q-1) / \log q$, whence

$$
(p-1) \log _{p}(n+1)<(q-1) \log _{q}(n+1), \quad n \in \mathbb{N} .
$$

Therefore, by Lemma 4 ,

$$
\max \left(\sigma_{p}(n), \sigma_{q}(n)\right) \leq(q-1) \log _{q}(n+1),
$$

and we find

$$
\frac{n-(q-1) \log _{q}(n+1)}{n-1}<\frac{n-\sigma_{p}(n)}{n-\sigma_{q}(n)}<\frac{n-1}{n-(q-1) \log _{q}(n+1)} .
$$

Notice that the function

$$
\gamma_{q}(x)=\frac{\log _{q} x}{x+q-2}
$$

decreases for $x>\max \left(q-2, e^{2}\right)$. Let $\alpha$ be the positive root of the equation $\log 2+\alpha / 2-\alpha^{2} / 8=\alpha \log q$. Then $\alpha<1$ and we have

$$
\log (2+\alpha)=\log 2+\log \left(1+\frac{\alpha}{2}\right)<\log 2+\frac{\alpha}{2}-\frac{\alpha^{2}}{4}+\frac{\alpha^{2}}{8}=\alpha \log q,
$$

i.e. $\log _{q}(2+\alpha)<\alpha$, and

$$
\gamma_{q}\left((2+\alpha) q^{2}\right)=\frac{2+\log _{q}(2+\alpha)}{(2+\alpha) q^{2}+q-2}<\frac{1}{q^{2}} .
$$

Therefore, for $n \geq(2+\alpha) q^{2}-1$ we have $\gamma_{q}(n+1)<1 / q^{2}$ and consequently $q \log _{q}(n+1)<(n+q-1) / q$. By $(20)$,

$$
\frac{n-\sigma_{p}(n)}{n-\sigma_{q}(n)}<\frac{n-1}{n-n / q-1+1 / q}=\frac{q}{q-1},
$$

and

$$
\frac{n-\sigma_{p}(n)}{n-\sigma_{q}(n)}>\frac{q-1}{q}>\frac{q-2}{q} .
$$

Thus

$$
q-2<(q-1) \frac{n-\sigma_{p}(n)}{n-\sigma_{q}(n)}<q .
$$

Further, since $n \in C_{p}^{!} \cap C_{q}^{!}$, there exist nonnegative integers $\alpha, \beta$ so that $e_{p}(n)=2^{\alpha}, e_{q}(n)=2^{\beta}$, where $\alpha \geq \beta$, and according to (15) we have $n-\sigma_{p}(n)=(p-1) 2^{\alpha}$ and $n-\sigma_{q}(n)=(q-1) 2^{\beta}$.

Consequently, by $(21)$ we obtain $q-2<(p-1) 2^{\alpha-\beta}<q$, whence $(p-1) 2^{\alpha-\beta}=q-1$, which is a contradiction. Therefore, $n<(2+\alpha) q^{2}-1$. 
It remains to notice that

$$
\begin{aligned}
\alpha & =2\left(\left((2 \log q-1)^{2}+2 \log 2\right)^{1 / 2}-(2 \log q-1)\right) \\
& =2(2 \log q-1)\left(\left(1+\frac{2 \log 2}{(2 \log q-1)^{2}}\right)^{1 / 2}-1\right) \\
& <2(2 \log q-1) \frac{\log 2}{(2 \log q-1)^{2}}=\frac{2 \log 2}{2 \log q-1},
\end{aligned}
$$

and the proposition follows.

Corollary 2. If $p=2$ and $q$ is a non-Fermat prime, then for $n \in$ $C_{2}^{!} \cap C_{q}^{!}$we have

$$
n \in\left(q, 2\left(1+\frac{\log 2}{2 \log q-1}\right) q^{2}-1\right) .
$$

In spite of the fact that the upper bound in Proposition 3 is quite convenient, the numerical experiments show that the value of the maximal element of the set $C_{p}^{!} \cap C_{q}^{!}$depends on the distance between $(q-1) /(p-1)$ and the nearest power of 2 (see Table 2). Now we shall give an estimate which is more sensitive to this factor.

Proposition 4. Under the conditions of Proposition 3, we have

$$
\frac{n}{\log (n+1)} \leq \max (a(p, q), b(p, q)) \quad \text { for } n \in C_{p}^{!} \cap C_{q}^{!},
$$

where

$$
\begin{aligned}
& a(p, q)=\frac{p-1}{\log p}\left(1-2^{-\left\{\log _{2} \frac{q-1}{p-1}\right\}}\right)^{-1}, \\
& b(p, q)=\frac{q-1}{\log q}\left(1-2^{\left\{\log _{2} \frac{q-1}{p-1}\right\}-1}\right)^{-1},
\end{aligned}
$$

and $\{x\}$ denotes the fractional part of $x$.

Proof. For $n \in C_{p}^{!} \cap C_{q}^{!}$there exist $\alpha \geq \beta, \alpha, \beta \in \mathbb{Z}_{+}$, so that $e_{p}(n)=2^{\alpha}$, $e_{q}(n)=2^{\beta}$, and by Corollary 1 we have

$$
\begin{aligned}
& \frac{n}{p-1}-\log _{p}(n+1) \leq 2^{\alpha}<\frac{n}{p-1}, \\
& \frac{n}{q-1}-\log _{q}(n+1) \leq 2^{\beta}<\frac{n}{q-1} .
\end{aligned}
$$

Therefore

$$
\frac{q-1}{p-1}\left(1-(p-1) \frac{\log _{p}(n+1)}{n}\right) \leq 2^{\alpha-\beta} \leq \frac{q-1}{p-1}\left(1-(q-1) \frac{\log _{q}(n+1)}{n}\right)^{-1},
$$


whence

$$
\begin{aligned}
& \alpha-\beta+\log _{2}(1-(q-1)\left.\frac{\log _{q}(n+1)}{n}\right) \leq \log _{2} \frac{q-1}{p-1} \\
& \leq \alpha-\beta+\log _{2}\left(1-(p-1) \frac{\log _{p}(n+1)}{n}\right) .
\end{aligned}
$$

Notice that

$$
b(p, q)>\frac{q-1}{\log q}\left(1-\frac{1}{2}\right)^{-1}=\frac{2(q-1)}{\log q} .
$$

Consequently, if $n / \log (n+1) \leq 2(q-1) / \log q$, then the conclusion of the proposition is satisfied trivially. Let now $n / \log (n+1)>2(q-1) / \log q$ (and certainly $n / \log (n+1)>2(p-1) / \log p)$. Then

$$
\begin{aligned}
& \log _{2}\left(1-(q-1) \frac{\log _{q}(n+1)}{n}\right)>-1 \\
& \log _{2}\left(1-(p-1) \frac{\log _{p}(n+1)}{n}\right)>-1 .
\end{aligned}
$$

By (22),

$$
\alpha-\beta-1<\log _{2} \frac{q-1}{p-1}<\alpha-\beta+1 .
$$

We distinguish between two cases:

CASE 1: $\left\lfloor\log _{2} \frac{q-1}{p-1}\right\rfloor=\alpha-\beta-1$. By the left inequality in (22) we have

$$
\alpha-\beta+\log _{2}\left(1-(q-1) \frac{\log _{q}(n+1)}{n}\right) \leq \alpha-\beta-1+\left\{\log _{2} \frac{q-1}{p-1}\right\},
$$

which implies $n / \log (n+1) \leq b(p, q)$, and the proposition follows.

CASE 2: $\left\lfloor\log _{2} \frac{q-1}{p-1}\right\rfloor=\alpha-\beta$. By the right inequality in (22) we have

$$
\alpha-\beta+\left\{\log _{2} \frac{q-1}{p-1}\right\} \leq \alpha-\beta-\log _{2}\left(1-(p-1) \frac{\log _{p}(n+1)}{n}\right),
$$

so that $n / \log (n+1) \leq a(p, q)$, and the proposition again follows.

\section{PROPOSITION 5.}

(i) If $p<q$ are primes such that $(q-1) /(p-1)$ is a power of 2 , then $n \in C_{p}^{!} \cap C_{q}^{!}$if $n \in C_{p}^{!}$and $\sigma_{p}(n)=\sigma_{q}(n)$. For $n \geq q^{2}$ the converse implication holds as well.

(ii) If $n \geq 4, p=2$ and $q$ is a Fermat prime, then $n \in C_{2}^{!} \cap C_{q}^{!}$if and only if $n \in C_{2}^{!}$and $\sigma_{2}(n)=\sigma_{q}(n)$.

Proof. (i) If $n \in C_{p}^{!}$and $\sigma_{p}(n)=\sigma_{q}(n)$ then by (15) there exists a nonnegative integer $\alpha$ such that

$$
n=(p-1) 2^{\alpha}+\sigma_{q}(n) .
$$


Moreover, by assumption, there exists $\gamma \in \mathbb{N}$ so that $(q-1) /(p-1)=2^{\gamma}$. Therefore, by (19) and (15),

$$
n=(q-1) 2^{\alpha-\gamma}+\sigma_{q}(n)=(q-1) e_{q}(n)+\sigma_{q}(n),
$$

and hence $e_{q}(n)=2^{\alpha-\gamma}$ and $n \in C_{p}^{!} \cap C_{q}^{!}$.

Conversely, if $n \in C_{p}^{!} \cap C_{q}^{!}$, then by (15) there exist nonnegative integers $\alpha$ and $\beta$ so that

$$
2^{\alpha}(p-1)+\sigma_{p}(n)=2^{\beta}(q-1)+\sigma_{q}(n)=n
$$

under the condition $(q-1) /(p-1)=2^{\gamma}$. Therefore, by $(23)$,

$$
\frac{n-\sigma_{p}(n)}{n-\sigma_{q}(n)}=2^{\alpha-\beta-\gamma} \text {. }
$$

Furthermore,

$$
1 \leq \sigma_{p}(n) \leq(p-1)\left\lfloor\log _{p}(n+1)\right\rfloor .
$$

Therefore, by (24)-(25),

$$
\frac{n-(p-1)\left\lfloor\log _{p}(n+1)\right\rfloor}{n-1} \leq 2^{\alpha-\beta-\gamma} \leq \frac{n-1}{n-(q-1)\left\lfloor\log _{q}(n+1)\right\rfloor} .
$$

Notice that the function $\left(\log _{p} x\right) / x$ decreases for $x>e$. Since $2 / p^{2} \leq$ $1 / 2(p-1)$ for $p \geq 2$, we have

$$
\frac{\log _{p}(n+1)}{n+1}<\frac{2}{p^{2}} \leq \frac{1}{2(p-1)} \quad \text { for } n>p^{2}-1,
$$

which yields

$$
(p-1)\left\lfloor\log _{p}(n+1)\right\rfloor<\frac{n+1}{2} .
$$

For $n>q^{2}-1$ we also have $(q-1)\left\lfloor\log _{q}(n+1)\right\rfloor<(n+1) / 2$, and by (26), $1 / 2<2^{\alpha-\beta-\gamma} \leq 2$. Now (24) implies $\sigma_{p}(n)=\sigma_{q}(n)$.

(ii) Let $p=2$ and $q$ be of the form $2^{k}+1$. Since $\sigma_{2}(n) \neq \sigma_{q}(n)$ for $n=4,5$, and $4,5 \notin C_{2}^{!}$, we suppose that $\max (6, q) \leq n<q^{2}$, and $n \in C_{2}^{!} \cap C_{q}^{!}$. By Proposition 1 there exists an $\alpha \geq 2$ so that $n=2^{\alpha}+i$, where $i=2$ or 3 . Since $\alpha \geq k$, put $\alpha=k+t, t \geq 0$. Notice that

$$
e_{q}(n)=\left\lfloor\frac{n}{q}\right\rfloor=\left\lfloor\frac{2^{k+t}+i}{2^{k}+1}\right\rfloor,
$$

where $i=2$ or 3 . For $t \geq 2$ we evidently have $2^{t-1}+1 \leq \frac{2^{k+t}+i}{2^{k}+1}<2^{t}$, which contradicts the fact that $n \in C_{q}^{!}$.

Therefore, consider $t=0$ and $t=1$ only. We obtain four numbers

$$
2^{k}+2=q+1, \quad 2^{k}+3=q+2, \quad 2^{k+1}+2=2 q, \quad 2^{k+1}+3=2 q+1,
$$

belonging to $C_{2}^{!} \cap C_{q}^{!}$, and for each of them $\sigma_{2}(n)=\sigma_{q}(n)$. 
Notice that for $n=3 \in C_{2}^{!} \cap C_{3}^{!}$, we have $\sigma_{2}(3) \neq \sigma_{3}(3)$.

As an important consequence we obtain the following statement.

Proposition 6. Let $q$ be a Fermat prime.

(i) For $\alpha \geq 2$, we have $2^{\alpha}+2 \in C_{q}^{!}$if and only if the diophantine equation

$$
q^{x}+q^{y}=2^{\alpha}+2
$$

is solvable in nonnegative integers $x, y$.

(ii) For $\alpha \geq 2$ we have $2^{\alpha}+3 \in C_{q}^{!}$if and only if the diophantine equation

$$
q^{x}+q^{y}+q^{z}=2^{\alpha}+3
$$

is solvable in nonnegative integers $x, y, z$.

Proof. Follows from Propositions 1 and 5.

Thus, to describe the set $C_{2}^{!} \cap C_{q}^{!}$for a Fermat prime $q$, it suffices to find all solutions of (28) in nonnegative integers.

4.2. Proof of Theorem 2 for non-Fermat primes. We start with several lemmas.

LEMMA 5. Let $q$ be an odd non-Fermat prime so that $2^{k-1}+3 \leq q<2^{k}$ for some $k \geq 3$. If some number $n$ of the form $n=2^{k+l}+i$, where $i=2$ or 3 , belongs to $C_{q}^{!}$, then $0 \leq l \leq k-1$. Now,

Proof. Notice that $l \geq 0$, as otherwise $n<q$ and $n \notin C_{q}^{!}$. Put $\Delta=2^{k}-q$.

$$
1 \leq \Delta \leq 2^{k}-2^{k-1}-3=2^{k-1}-3 \leq q-6
$$

Furthermore,

$$
n=2^{k+l}+i=2^{l}\left(2^{k}-\Delta\right)+2^{l} \Delta+i=2^{l} q+\left(2^{l} \Delta+i\right) .
$$

Since, by Proposition $1, n \in C_{2}^{!}$, we have $n \in C_{2}^{!} \cap C_{q}^{!}$, and, by Corollary 1 , $n \leq 3 q^{2}-1$. Therefore,

$$
e_{q}(n)=\left\lfloor\frac{n}{q}\right\rfloor+\left\lfloor\frac{n}{q^{2}}\right\rfloor \leq\left\lfloor\frac{n}{q}\right\rfloor+2 .
$$

Suppose that $l \geq k$. Then $q<2^{k} \leq 2^{l}$, and by (30),

$$
e_{q}(n) \geq\left\lfloor\frac{n}{q}\right\rfloor \geq 2^{l}+1 \text {. }
$$

On the other hand, by (29)-(31) we have

$$
\begin{aligned}
& e_{q}(n) \leq 2^{l}+\left\lfloor\frac{2^{l} \Delta+3}{q}\right\rfloor+2 \leq 2^{l}+2+\left\lfloor\frac{2^{l}\left(2^{k-1}-3\right)+3}{q}\right\rfloor \\
& \quad<2^{l}+2+\left\lfloor 2^{l}-\frac{3 \cdot 2^{l}}{2^{k-1}+3}\right\rfloor \leq 2^{l+1}+2-\frac{3 \cdot 2^{k}}{2^{k-1}+2^{k-1}}=2^{l+1}-1 .
\end{aligned}
$$

Now (32)-(33) contradict the condition $n \in C_{q}^{!}$. Thus, $l \leq k-1$. 
Lemma 6. Under the conditions of Lemma 5, the number $n=2^{k+l}+i$, where $i=2$ or 3 , belongs to $C_{q}^{!}$if and only if $2^{l} \Delta+i<q$.

Proof. If $2^{l} \Delta+i<q$, then by (30),

$$
e_{q}(n)=2^{l}+\left\lfloor\frac{2^{l}}{q}\right\rfloor=2^{l}
$$

i.e., $n \in C_{q}^{!}$.

Conversely, let $n \in C_{q}^{!}$. We distinguish between two cases: Hence

CASE a: $l=k-1$. We have $n=2^{2 k-1}+i \in C_{2}^{!} \cap C_{q}^{!}$, where $i=2$ or 3 .

$$
\left\lfloor\frac{n}{q^{2}}\right\rfloor \leq\left\lfloor\frac{2^{2 k-1}+3}{\left(2^{k-1}+3\right)^{2}}\right\rfloor \leq 1 .
$$

By (29), (30) and (34),

$$
e_{q}(n) \leq 2^{k-1}+\left\lfloor\frac{2^{k-1} \Delta+i}{q}\right\rfloor+1 \leq 2^{k-1}+\left\lfloor\frac{2^{k-1}(q-6)+i}{q}\right\rfloor+1 .
$$

Since $q \leq 2^{k}-1$, and the function $\left(2^{k-1}(x-6)+i\right) / x$ increases,

$$
\frac{2^{k-1}(q-6)+i}{q} \leq \frac{2^{k-1}\left(2^{k}-7\right)+3}{2^{k}-1}<2^{k-1}-1 .
$$

Therefore, by (35),

$$
e_{q}(n) \leq 2^{k-1}+2^{k-1}-2+1=2^{k}-1 .
$$

On the other hand, by (30),

$$
e_{q}(n) \geq 2^{k-1}+\left\lfloor\frac{2^{k-1} \Delta+i}{q}\right\rfloor .
$$

If $2^{k-1} \Delta+i \geq q$, then (36)-(37) imply $2^{k-1}+1 \leq e_{q}(n) \leq 2^{k}-1$, which contradicts the condition $n \in C_{q}^{!}$.

CASE b: $l \leq k-2$. Now $n \leq 2^{2 k-2}+3$, and instead of (32) we obtain $\left\lfloor n / q^{2}\right\rfloor=0$, and therefore instead of (35) we find that

$$
e_{q}(n) \leq 2^{l}+\left\lfloor\frac{2^{l}(q-6)+3}{q}\right\rfloor \leq 2^{l}+\left(2^{l}-1\right)=2^{l+1}-1 .
$$

On the other hand, by (30),

$$
e_{q}(n)=2^{l}+\left\lfloor\frac{2^{l} \Delta+i}{q}\right\rfloor
$$

and again we conclude that, if $2^{l} \Delta+i \geq q$, then (38)-(39) contradict the condition $n \in C_{q}^{!}$.

Now we need an additional technical lemma. 


\section{Lemma 7. The diophantine equation}

$$
n\left(2^{m}+1\right)=2^{k+m}+3, \quad m \geq 0, k \geq 0, n \in \mathbb{N},
$$

has only the following solutions:

1) $k=0, m=0, n=2$.

2) $k \equiv 3(\bmod 4), m=2, n=\left(2^{k+2}+3\right) / 5$.

Proof. Let

$$
k=r m+s, \quad 0 \leq s<m .
$$

It is easy to see that

$$
\left(2^{m}+1\right) \sum_{i=0}^{\lfloor k / m\rfloor}(-1)^{i} 2^{k-m i}=2^{k+m}+(-1)^{r} 2^{s}, \quad m \geq 1 .
$$

Therefore,

$$
2^{k+m}+3 \equiv(-1)^{r+1} 2^{s}+3\left(\bmod 2^{m}+1\right), \quad m \geq 1 .
$$

If (40) is valid, then by (41),

$$
(-1)^{r+1} 2^{s}+3 \equiv 0\left(\bmod 2^{m}+1\right) .
$$

If $r$ in $(42)$ is even, then $2^{s}-3 \equiv 0\left(\bmod 2^{m}+1\right)$. This is impossible, since $2^{s}-3<2^{m}+1$ and $2^{5}-3 \neq 0$. If the $r$ is odd, then $2^{s}+3 \equiv 0\left(\bmod 2^{m}+1\right)$, $m \geq 1$. If here $m \geq 3$, then $2^{s}+3 \leq 2^{m-1}+3<2^{m}+1$. Therefore, $m \leq 2$, and in addition the case $m=1$ is impossible. If $m=2$, from (40) we find $n=\left(2^{k+2}+3\right) / 5$. Also notice that if in (40) we have $m=0$, then $2 n=2^{k}+3$, so that $k=0$ and $n=2$.

Now we are able to complete the proof of Theorem 2 in the case of a non-Fermat prime $q$.

1) Let $q=2^{k-1}+3$. First suppose that $k \geq 4$, i.e., $q \geq 11$. Then by Lemma 6 , we have $2^{k+l}+i \in C_{2}^{!} \cap C_{q}^{!}, i=2,3, l \geq 0$, if and only if $\Delta \cdot 2^{l}+i<q=2^{k-1}+3$, where $\Delta=2^{k-1}-3$. Thus,

$$
\left(2^{k-1}-3\right) 2^{l} \leq 2^{k-1}+2-i, \quad i=2,3 .
$$

If $l \geq 1$, this is impossible for $k \geq 4$. Therefore $l=0$ and we have two elements from $C_{2}^{!} \cap C_{q}^{!}$, namely

$$
2^{k}+2=2 q-4 \quad \text { and } \quad 2^{k}+3=2 q-3 .
$$

Moreover, in this case $q=2^{k-1}+3 \in C_{2}^{!} \cap C_{q}^{!}$. Thus, if $k \geq 4$,

$$
C_{2}^{!} \cap C_{q}^{!}=\{q, 2 q-4,3 q-3\} .
$$

It remains to deal with the case $q=7, k=3$. Here (43) is satisfied if $2^{l} \leq 6-i$, i.e., $l=0,1$ for $i=2,3$ and $l=2$ for $i=2$. That gives five numbers from $C_{2}^{!} \cap C_{7}^{!}: 10,11,18,19$ and 34 . Moreover, $7 \in C_{2}^{!} \cap C_{7}^{!}$. Thus $\left|C_{2}^{!} \cap C_{7}^{!}\right|=6$. 
As a simple consequence we obtain the following statement.

Proposition 7.

$$
C^{!}=\{1,2,3,6,7,10,11\}
$$

Proof. It is sufficient to consider the numbers $1,2,3,4,5,6,7,10,11$, $18,19,34$.

2) Let $q=\left(2^{k+2}+3\right) / 5$, where $k \equiv 3(\bmod 4)$. The smallest $q>7$ of this kind is $q=103$, obtained for $k=7$. The smallest $q>103$ is $q=6710887$, obtained for $k=23$. Let $k \geq 7$. Here

$$
\Delta=2^{k}-\frac{2^{k+2}+3}{5}=\frac{2^{k}-3}{5} .
$$

Now by Lemma 6 we have $2^{k+l}+i \in C_{2} \cap C_{q}, i=2,3, l \geq 0$, if and only if

$$
\Delta \cdot 2^{l}+i=\frac{2^{k+l}-3 \cdot 2^{l}+5 i}{5}<q=\frac{2^{k+2}+3}{5}, \quad k \geq 7 .
$$

Hence $l \leq 2$, and in the case $l=2$,

$$
2^{k+2}+i<2^{k+2}+3
$$

and $i=2$. Thus we have exactly five suitable numbers:

$$
\begin{array}{ll}
l=0: & n_{1}=2^{k}+2=\frac{5 q+5}{4}, \quad n_{2}=2^{k}+3=\frac{5 q+9}{4}, \\
l=1: & n_{3}=2^{k}+2=\frac{5 q+1}{2}, \quad n_{4}=2^{k+1}+3=\frac{5 q+3}{2}, \\
l=2: & n_{5}=2^{k+2}+2=5 q-1,
\end{array}
$$

so that $\left|C_{2}^{!} \cap C_{q}^{!}\right|=5$.

3) Let $q \geq 2^{k-1}+5$ and $q \neq\left(2^{k+2}+3\right) / 5$. First of all, we will show that in this case $\left|C_{2}^{!} \cap C_{q}^{!}\right|$is even. To this end, it suffices to show that the inequality $\Delta \cdot 2^{l}+2<q$ implies $\Delta \cdot 2^{l}+3<q$. Indeed, if $\Delta \cdot 2^{l}+3=q$ or $\left(2^{k}-q\right) \cdot 2^{l}+3=q$, then

$$
q\left(2^{l}+1\right)=2^{k+l}+3
$$

and by Lemma 7 we have $l=2, q=\left(2^{k+2}+3\right) / 5$, where $k \equiv 3(\bmod 4)$. This contradicts the assumption. Therefore, by Lemma $6,2^{k+l}+i \in C_{2}^{!} \cap C_{q}^{!}$ if and only if $\left(2^{k}-q\right) 2^{l}+3<q$. Notice that $l=0$ is trivially a suitable case, which gives two numbers from $C_{2}^{!} \cap C_{q}^{!}$, namely, $2^{k}+2$ and $2^{k}+3$. In the case of $l \geq 1$, the inequality $\left(2^{k}-q\right) 2^{l}+3<q$ implies $\left(2^{k}-q\right) 2^{l} \leq q-5$. Thus, $n \in C_{2}^{!} \cap C_{q}^{!}$if and only if $n=2^{k+l}+i$, where $i=2$ or 3 , and $0 \leq l \leq\left\lfloor\log _{2} \frac{q-5}{2^{k}-q}\right\rfloor$. Therefore

$$
\left|C_{2}^{!} \cap C_{q}^{!}\right|=2\left(1+\left\lfloor\log _{2} \frac{q-5}{2^{k}-q}\right\rfloor\right) .
$$

This completes the proof of Theorem 2 when $q$ is a non-Fermat prime. 
4.3. Proof of Theorem 2 when $q$ is a Fermat prime. According to Proposition 6 , we need to investigate the diophantine equation

$$
2^{\alpha}+3=q^{x}+q^{y}+q^{z}
$$

in nonnegative integers $\alpha, x, y, z$, where $q$ is a Fermat prime.

We need the following result of G. C. Gerono (1871).

Proposition 8 (see [15, p. 374]). The diophantine equation $2^{\alpha}+1=g^{x}$ for $\alpha \geq 2, x \geq 2, g \geq 2$, has the only solution $\alpha=3, x=2, g=3$.

Taking into account this result, we break up the investigation of (45) into the following cases:

1. $q \geq 17, x, y, z \geq 1$,

2. $q \geq 17, z=0, x \neq y$

(except the solution $\alpha=1+\log _{2}(q-1), x=y=1$ ),

3. $q=3, z=0, x \neq y$

(except the solutions $\alpha=2, x=y=1 ; \alpha=3, x=y=2$ ),

4. $q=5, z=0, x \neq y$ (except the solution $\alpha=3, x=y=1$ ),

5. $q=5, x, y, z \geq 1$.

4.3.1. CASE 1: $q \geq 17, x, y, z \geq 1$. We start with the following straightforward lemma.

LEMMA 8. If $q=2^{2^{h-1}}+1, h \geq 3$, then the subgroup of $(\mathbb{Z} / q \mathbb{Z})^{*}$ generated by 2 is

$$
\left\{2^{j}: 0 \leq j \leq 2^{h-1}\right\} \cup\left\{q-2^{j}: 1 \leq j \leq 2^{h-1}\right\} .
$$

Proposition 9. If $q \geq 17$ is a Fermat prime, then (45) has no solutions with $x, y, z \geq 1$.

Proof. It is sufficient to prove that $2^{\alpha}+3$ is not divisible by $q$. Let $q=2^{2^{h-1}}+1, h \geq 3$. If $2^{m}+3 \equiv 0(\bmod q)$ then -3 is generated by 2 in $(\mathbb{Z} / q \mathbb{Z})^{*}$. Using Lemma 8 , we easily see that the only possibility is $h=2$.

4.3.2. CASE 2: $q \geq 17, z=0, x \neq y$.

Lemma 9. If $q=2^{2^{h-1}}, h \geq 3$, is a Fermat prime, then $q$ divides $2^{m}+1$ if and only if $m=2^{h} k-2^{h-1}$ for some $k \in \mathbb{N}$.

Proof. Follows from the connection between $j$ and $2^{j}(\bmod m)$, which is implicit in Lemma 8.

Suppose now that in (45) we have $z=0, x, y \geq 1, x \neq y, q=2^{2^{h-1}}+1$, $h \geq 3$. Then $q \mid 2^{\alpha-1}+1$, and by Lemma 9 we have

$$
\alpha=2^{h}(k-1)+2^{h-1}+1
$$

for some $k \geq 1$, so that

$$
2\left(2^{2^{h} k-2^{h-1}}+1\right)=\left(2^{2^{h-1}}+1\right)^{x}+\left(2^{2^{h-1}}+1\right)^{y} .
$$


If $k=1$, then

$$
2=\left(2^{2^{h-1}}+1\right)^{x-1}+\left(2^{2^{h-1}}+1\right)^{y-1},
$$

whence $x=y=1$, contradicting the assumption $x \neq y$. Hence $k \geq 2$. Consequently, $2^{h} k-2^{h-1} \geq 2^{h+1}-2^{h-1}>2^{h}$, so that (46) yields

$$
2 \equiv 2^{2^{h-1}} x+2^{2^{h-1}} y+2\left(\bmod 2^{2^{h}}\right) .
$$

Thus

$$
x+y \equiv 0\left(\bmod 2^{2^{h-1}}\right),
$$

and in particular $x$ and $y$ have the same parity.

Suppose that, in (46), $x$ and $y$ are both even. Taking (46) modulo 3, we obtain

$$
\begin{aligned}
2 & \neq\left(2^{2^{h-1}}+1\right)^{x}+\left(2^{2^{h-1}}+1\right)^{y} \\
& =\left(\left(2^{2^{h-1}}+2\right)-1\right)^{x}+\left(\left(2^{2^{h-1}}+2\right)-1\right)^{y} \\
& =\left(2\left(2^{2^{h-1}}+1\right)-1\right)^{x}+\left(2\left(2^{2^{h-1}}+1\right)-1\right)^{y} \equiv 2(\bmod 3),
\end{aligned}
$$

which is a contradiction. Thus, $x$ and $y$ are both odd. Let $x=2 l-1$, $y=2 m-1, l, m \in \mathbb{N}$. By $(47)$,

$$
l+m \equiv 1\left(\bmod 2^{2^{h-1}-1}\right) .
$$

Hence, $l$ and $m$ have different parities. Moreover, by (46),

$$
2\left(2^{2^{h} k-2^{h-1}}+1\right)\left(2^{2^{h-1}}+1\right)=\left(\left(2^{2^{h-1}}+1\right)^{2}\right)^{l}+\left(\left(2^{2^{h-1}}+1\right)^{2}\right)^{m} .
$$

Notice that, since $h \geq 3$, we have $2^{2^{h-1}}+1=\left(2^{4}\right)^{2^{h-3}}+1 \equiv 2(\bmod 5)$. Therefore $\left(2^{2^{h-1}}+1\right)^{2} \equiv-1(\bmod 5)$, and according to $(48)$, since $l$ and $m$ have different parities, we have $0 \equiv 2^{2^{h} k-2^{h-1}}+1=2^{2^{h-1}(2 k-1)}+1=$ $\left(\left(2^{4}\right)^{2^{h-3}}\right)^{2 k-1}+1 \equiv 2(\bmod 5)$. This contradiction shows that $(45)$ has no solutions with $z=0, x \geq 1, y \geq 1$ and $x \neq y$. A simple sorting out of other possibilities gives the following.

Proposition 10. If $q \geq 17$ is a Fermat prime then (45) has only the following solutions (up to permutation of $x, y, z$ ):

- $x=1, y=0, z=0, \alpha=\log _{2}(q-1)$;

- $x=y=1, z=0, \alpha=\log _{2}(q-1)$.

4.3.3. CASE 3: $q=3, z=0, x \neq y$. Here we investigate the equation

$$
2^{\alpha}+2=3^{x}+3^{y} \text {. }
$$

Since

$$
2^{\alpha-1}+1=(3-1)^{\alpha-1}+1 \equiv \begin{cases}0(\bmod 3), & \alpha \equiv 0(\bmod 2), \\ 2(\bmod 3), & \alpha \equiv 1(\bmod 2),\end{cases}
$$


$\alpha$ in (49) is even: $\alpha=2 t, t \geq 2$. Rewrite (49) as

$$
4^{t}+2=3^{x}+3^{y}, \quad t \geq 2, x, y \geq 1, x \neq y .
$$

Suppose that, say, $x>y$. Suppose $x, y$ have distinct parities. Then

$$
3^{x}+3^{y}=3^{y}\left(3 \cdot 3^{x-y-1}+1\right) \equiv 0(\bmod 4),
$$

which contradicts (50). Next, suppose $x, y$ are both odd. Then $3^{x}+3^{y}-2=3\left(3^{x-1}+3^{x-1}\right)-2=3\left(9^{(x-1) / 2}+9^{(x-1) / 2}\right)-2 \equiv 4(\bmod 8)$, which is again impossible in (50). It follows that $x, y$ are both even, say, $x=2 r, y=2 u$. Substitute this in (50):

$$
4^{t}+2=9^{r}+9^{u} \quad(r>u \geq 1, t \geq 2) .
$$

Writing $t=3 s+i$, where $s \geq 0$ and $i=1,2,3$, we easily obtain $2^{2 i-1}+1 \equiv 0$ $(\bmod 9)$. Thus $i=2$ and $t=3 s+2$, and instead of $(51)$ we consider the equation

$$
2^{6 s+4}+2=9^{r}+9^{u}, \quad r>u \geq 1, s \geq 0
$$

whence

$$
4 \equiv 2^{r}+2^{u}(\bmod 7)
$$

Since $2^{1} \equiv 2,2^{2} \equiv 4,2^{3} \equiv 1(\bmod 7)$, it follows that $r \equiv u \equiv 1(\bmod 3)$.

Put $r=3 v+1, u=3 w+1, v>w \geq 0$. Then by (52),

$$
4^{3 s+2}+2=9^{3 v+1}+9^{3 w+1}, \quad v>w \geq 0, s \geq 0 .
$$

By taking this modulo 5 we have

$$
(-1)^{s}+2 \equiv(-1)^{v+1}+(-1)^{w+1}(\bmod 5) .
$$

Therefore, $v$ and $w$ have the same parity; moreover, $v$ and $w$ are even, and $s$ is also even.

Put $s=2 \sigma, v=2 \nu, w=2 \omega$. Then by (53),

$$
4^{6 \sigma+2}+2=9^{6 \nu+1}+9^{6 \omega+1}, \quad \nu>\omega \geq 0 .
$$

Let us write (54) in the form

$$
16 \cdot 8^{4 \sigma}+2=9\left(27^{4 \nu}+27^{4 \omega}\right), \quad \nu>\omega \geq 0 .
$$

Now by taking this modulo 19 we have

$$
-3 \cdot 8^{4 \sigma}+2 \equiv 9\left(8^{4 \nu}+8^{4 \omega}\right)(\bmod 19) .
$$

Taking into account that

$$
8^{4}=64^{2} \equiv 7^{2} \equiv 11(\bmod 19),
$$

we have

$$
9\left(11^{\nu}+11^{\omega}\right)+3 \cdot 11^{\sigma} \equiv 2(\bmod 19) .
$$

Since $11^{1} \equiv 11,11^{2} \equiv 7,11^{3} \equiv 1 ; 9 \cdot 11^{1} \equiv 4,9 \cdot 11^{2} \equiv 6,9 \cdot 11^{3} \equiv 9$; $3 \cdot 11^{1} \equiv 14,3 \cdot 11^{2} \equiv 2,3 \cdot 11^{3} \equiv 3(\bmod 19)$, we see that the unique 
possibility in $(56)$ is $\sigma \equiv \nu \equiv \omega \equiv 0(\bmod 3)$. Finally, put $\nu=3 \beta, \omega=3 \gamma$, $\sigma=3 \delta, \beta>\gamma \geq 0$. By (54) we have

$$
2^{36 \delta+4}+2=3^{36 \beta+2}+3^{36 \gamma+2}, \quad \beta>\gamma \geq 0 .
$$

Consider two cases:

1) $\gamma=0, \beta \geq 1$. Then

$$
2^{36 \delta+4} \equiv 7(\bmod 27), \quad \text { i.e. } \quad 16 \cdot 4^{\varphi(27) \delta} \equiv 7(\bmod 27),
$$

whence $16 \equiv 7(\bmod 27)$. We have a contradiction.

2) $\gamma \geq 1, \beta \geq 2$. Then

$$
2^{36 \delta+4} \equiv 25(\bmod 27), \quad \text { i.e. } \quad 16 \cdot 4^{\varphi(27) \delta} \equiv 25(\bmod 27),
$$

whence $16 \equiv 25(\bmod 27)$. Again we have a contradiction. Consequently, we have proved the following statement.

Proposition 11. The diophantine equation (49): $2^{\alpha}+2=3^{x}+3^{y}$ does not have solutions in $x \geq 1, y \geq 1, x \neq y$.

By a simple sorting out of the possibilities not included in Proposition 11, using Proposition 8, we obtain the following consequence.

Corollary 3. If $x, y \geq 0, \alpha \geq 2$ then the diophantine equation $2^{\alpha}+2=$ $3^{x}+3^{y}$ has only the following solutions:

- $\alpha=4, x=y=2$;

- $\alpha=3, x=2, y=0 ; \alpha=3, x=0, y=2$;

- $\alpha=2, x=y=1$.

4.3.4. CASE 4: $q=5, z=0, x \neq y$. Here we investigate the equation

$$
2^{\alpha}+2=5^{x}+5^{y}, \quad x, y \geq 1, x \neq y, \alpha \geq 5 .
$$

(It is evident that for $\alpha \leq 4,(57)$ has no solutions in $x \geq 1, y \geq 1, x \neq y$.) Since modulo 5 we have $2^{1} \equiv 2,2^{2} \equiv 4,2^{3} \equiv 3,2^{4} \equiv 1$, it follows that in (57), $\alpha=4 k+3, k \geq 1$. Thus by (57),

$$
2^{4 k+3}+2=5^{x}+5^{y}, \quad k \geq 1, x \geq 1, y \geq 1, x \neq y .
$$

By taking this modulo 16 we have

$$
2 \equiv(4+1)^{x}+(4+1)^{y} \equiv(x+y)+2(\bmod 16) .
$$

Therefore, $x+y \equiv 0(\bmod 4)$, and $x, y$ have the same parity. Taking now (58) modulo 3, we have

$$
1 \equiv(6-1)^{x}+(6-1)^{y} \equiv 2(-1)^{x}(\bmod 3) .
$$

Consequently, both $x$ and $y$ are odd. 
Put $x=2 l-1, y=2 m-1$. Then $l+m \equiv 1(\bmod 2)$. Now instead of (58) we have

$$
\begin{aligned}
2^{4 k+3}+2=5^{2 l-1}+5^{2 m-1}, \quad k \geq 1, l & \geq 1, m \geq 1, \\
& l \neq m, l+m \equiv 1(\bmod 2),
\end{aligned}
$$

i.e. $5\left(2^{4 k+3}+2\right)=25^{l}+25^{m}$. By taking this modulo 13 we immediately obtain

$$
2^{4 k+2}+1 \equiv 0, \quad \text { i.e. } \quad 4^{2 k+1} \equiv 12,4^{2 k} \equiv 3(\bmod 13), k \geq 1 .
$$

Since modulo 13 we have

$$
4^{1} \equiv 4, \quad 4^{2} \equiv 3, \quad 4^{3} \equiv 12, \quad 4^{4} \equiv 9, \quad 4^{5} \equiv 10, \quad 4^{6} \equiv 1,
$$

from (60) we obtain

$$
2 k \equiv 2(\bmod 6), \quad \text { i.e. } \quad k \equiv 1(\bmod 3) .
$$

Put $k=3 r+1, r \geq 0$. Then by (59),

$$
2^{12 r+7}+2=5^{2 l-1}+5^{2 m-1}, \quad l \geq 1, m \geq 1, l \neq m, l+m \equiv 1(\bmod 2) .
$$

Further, by taking this modulo 7 we have

$$
4 \equiv-2^{2 l-1}-2^{2 m-1}, \quad \text { i.e. } \quad 4^{l}+4^{m} \equiv 6(\bmod 7) .
$$

Since $4^{1} \equiv 4,4^{2} \equiv 2,4^{3} \equiv 1(\bmod 7)$, it follows that either $l \equiv 1, m \equiv 2$ $(\bmod 3)$ or $l \equiv 2, m \equiv 1(\bmod 3)$. By symmetry it suffices to consider only the first possibility. Put $l=3 u+1, m=3 v+2, u, v \geq 0$. Since in (61), $l+m \equiv 1(\bmod 2)$, we have $u+v \equiv 0(\bmod 2)$, and by $(61)$ we obtain

$$
2^{12 r+7}+2=5^{6 u+1}+5^{6 v+3}, \quad r, u, v \geq 0, u+v \equiv 0(\bmod 2) .
$$

We shall write (62) in the form

$$
2^{12 r+7}+2=5 \cdot 125^{2 u}+125^{2 v+1} .
$$

Since $125 \equiv-8(\bmod 19)$, we have

$$
2^{12 r+7}+2 \equiv 5 \cdot 2^{6 u}-2^{6 v+3}(\bmod 19) .
$$

Since $2^{6} \equiv 7(\bmod 19)$, we obtain $2 \cdot 7^{2 r+1}+2 \equiv 5 \cdot 7^{u}-8 \cdot 7^{v}(\bmod 19)$, i.e. (63) $7^{2 r+1}+7^{u+1}+4 \cdot 7^{v} \equiv 18(\bmod 19), \quad r, u, v \geq 0, u+v \equiv 0(\bmod 2)$.

Notice that modulo 19 we have $7^{1} \equiv 7,7^{2} \equiv 11,7^{3} \equiv 1 ; 4 \cdot 7^{1} \equiv 9,4 \cdot 7^{2} \equiv 6$, $4 \cdot 7^{3} \equiv 4$. Consequently, as is easy to check, we have only three cases in (63):

(a) $2 r+1 \equiv 0, u \equiv 1, v \equiv 2(\bmod 3)$,

(b) $2 r+1 \equiv 2, u \equiv 2, v \equiv 2(\bmod 3)$,

(c) $2 r+1 \equiv 1, u \equiv 0, v \equiv 0(\bmod 3)$.

CASE (a). Put $2 r+1=3(2 \lambda+1)$, i.e. $r=3 \lambda+1, u=3 \xi+1, v=3 \eta+2$, $\lambda, \xi, \eta \geq 0$. 
Since $u+v \equiv 0(\bmod 2)$, we have $\xi+\eta \equiv 1(\bmod 2)$. By $(62)$,

$$
2^{36 \lambda+19}+2=5^{18 \xi+7}+5^{18 \eta+15} .
$$

By taking this modulo 125 we have $2^{36 \lambda+18} \equiv-1(\bmod 125)$.

Since $\min \left\{\alpha \geq 1: 2^{\alpha} \equiv-1(\bmod 125)\right\}=50$ and $\varphi(125)=100$ we have

$$
36 \lambda+18 \equiv 50(\bmod 100), \quad \text { i.e. } \quad 9 \lambda \equiv 8(\bmod 25) .
$$

On the other hand, notice that $5^{6}+1=15626=2 \cdot 13 \cdot 601$ and $5^{6} \equiv-1$ $(\bmod 601)$. Now by $(64)$ we have

$$
2^{36 \lambda+19}+2=5\left(5^{6}\right)^{3 \xi+1}+125\left(5^{6}\right)^{3 \eta+2} \equiv 5(-1)^{\xi+1}+125(-1)^{\eta}(\bmod 601) .
$$

Taking into account that $\xi+\eta \equiv 1(\bmod 2)$, we conclude that, modulo 601,

$$
2^{36 \lambda+19}+2 \equiv \begin{cases}130, & \eta \text { is even } \\ -130, & \eta \text { is odd }\end{cases}
$$

i.e.

$$
2^{36 \lambda+18} \equiv \begin{cases}64, & \eta \text { is even } \\ -66, & \eta \text { is odd }\end{cases}
$$

Now notice that all residues of powers of 2 modulo 601 are:

$$
\begin{aligned}
& 2^{i}, i=1,2, \ldots, 9 ; 2^{10} \equiv 423,2^{11} \equiv 245,2^{12} \equiv 490,2^{13} \equiv 379, \\
& 2^{14} \equiv 157, \quad 2^{15} \equiv 314, \quad 2^{16} \equiv 27, \quad 2^{17} \equiv 54, \\
& 2^{18} \equiv 108, \quad 2^{19} \equiv 216, \quad 2^{20} \equiv 432, \quad 2^{21} \equiv 263, \\
& 2^{22} \equiv 526, \quad 2^{23} \equiv 451, \quad 2^{24} \equiv 301, \quad 2^{25} \equiv 1 .
\end{aligned}
$$

Now we see that in $(66)$ the residue $-66 \equiv 535(\bmod 601)$ is impossible. Therefore $\eta$ is even and $2^{36 \lambda+12} \equiv 1(\bmod 601)$, so $36 \lambda+12 \equiv 0(\bmod 25)$, hence $9 \lambda \equiv-3(\bmod 25)$. That contradicts $(65)$.

Case (b). Put $2 r+1=3(2 \lambda+1)+2$, i.e. $r=3 \lambda+2, u=3 \xi+2$, $v=3 \eta+2, \lambda, \xi, \eta \geq 0$. Since $u+v \equiv 0(\bmod 2)$, we have $\xi+\eta \equiv 0(\bmod 2)$. By (62),

$$
2^{36 \lambda+31}+2=5^{18 \xi+13}+5^{18 \eta+15} .
$$

By taking this modulo 125 we have $2^{36 \lambda+30} \equiv-1(\bmod 125)$. Consequently, as in Case (a) we obtain

$$
36 \lambda+30 \equiv 50(\bmod 100), \quad \text { i.e. } \quad 9 \lambda \equiv 5(\bmod 25) .
$$

On the other hand, again, since $5^{6} \equiv-1(\bmod 601)$, by $(67)$ we have

$$
2^{36 \lambda+31}+2=5\left(5^{6}\right)^{3 \xi+2}+125\left(5^{6}\right)^{3 \eta+2} \equiv 5(-1)^{\xi}+125(-1)^{\eta}(\bmod 601) .
$$

Taking into account that $\xi+\eta \equiv 0(\bmod 2)$, we conclude that

$$
2^{36 \lambda+31}+2 \equiv \begin{cases}130, & \eta \text { is even } \\ -130, & \eta \text { is odd }\end{cases}
$$


and as in Case (a) we find that $\eta$ is even and

$$
2^{36 \lambda+24} \equiv 64(\bmod 601), \quad 36 \lambda+24 \equiv 0(\bmod 25),
$$

i.e. $9 \lambda \equiv-6(\bmod 25)$. That contradicts $(68)$.

CASE (c). Put $2 r+1=3(2 \lambda)+1$, i.e. $r=3 \lambda$, and $u=3 \xi, v=3 \eta$, $\lambda, \xi, \eta \geq 0$. Since $u+v \equiv 0(\bmod 2)$, we have $\xi+\eta \equiv 0(\bmod 2)$. By $(62)$,

$$
2^{36 \lambda+7}+2=5^{18 \xi+1}+5^{18 \eta+3} .
$$

Here we consider two subcases: $\left(\mathrm{c}^{\prime}\right) \xi \geq 1,\left(\mathrm{c}^{\prime \prime}\right) \xi=0$.

Subcase $\left(c^{\prime}\right): \xi \geq 1$. By taking (69) modulo 125 we find

$$
2^{36 \lambda+6} \equiv-1(\bmod 125) \text {. }
$$

Therefore, as above $36 \lambda+6 \equiv 50(\bmod 100)$ and so

$$
9 \lambda \equiv 11(\bmod 25) \text {. }
$$

On the other hand, by (69) we have

$$
2^{36 \lambda+7}+2=5\left(5^{6}\right)^{3 \xi}+125\left(5^{6}\right)^{3 \eta} \equiv 5(-1)^{\xi}+125(-1)^{\eta}(\bmod 601)
$$

and

$$
2^{36 \lambda+7}+2 \equiv \begin{cases}130, & \eta \text { is even } \\ -130, & \eta \text { is odd }\end{cases}
$$

As above we find that $\eta$ is even and $2^{36 \lambda} \equiv 1(\bmod 601)$. Consequently, $36 \lambda \equiv 0(\bmod 25)$. This contradicts $(70)$.

SubCase $\left(c^{\prime \prime}\right): \xi=0$. By $(69)$,

$$
2^{36 \lambda+7}-3=5^{18 \eta+3}
$$

This equation is a special case of the following equation considered in [1]:

$$
1+2^{a}=3^{b} 5^{c}+2^{d} 3^{e} 5^{f}
$$

for $a=36 \lambda+7, b=0, c=18 \eta+3, d=2, e=f=0$. Since $a \geq 7, c \geq 3$, by [1] the equation (71) has only the solution $\lambda=0, \eta=0$.

Thus, we proved

Proposition 12. The diophantine equation (57): $2^{\lambda}+2=5^{x}+5^{y}$ has only the following solution: $\lambda=7, x=3, y=1$, in $\lambda \geq 2, x>y \geq 1$.

4.3.5. CASE 5: $q=5, x, y, z \geq 1$. Here we investigate the equation

$$
2^{\alpha}+3=5^{x}+5^{y}+5^{z}, \quad x, y, z \geq 1, \alpha \geq 4 .
$$

Since in $(72), 2^{\alpha} \equiv 2(\bmod 5)$, we obtain $\lambda=4 t+1, t \geq 1$. Thus,

$$
2^{4 t+1}+3=5^{x}+5^{y}+5^{z}, \quad t, x, y, z \geq 1 .
$$

By taking this modulo 3 we have

$$
2 \equiv(-1)^{x}+(-1)^{y}+(-1)^{z}(\bmod 3) .
$$


By symmetry, it is sufficient to consider the case: $x \equiv 0, y \equiv z \equiv 1(\bmod 2)$. Put $x=2 k, y=2 l-1, z=2 m-1$. Then by (73),

$$
2^{4 t+1}+3=5^{2 k}+5^{2 l-1}+5^{2 m-1}, \quad t, k, l, m \geq 1 .
$$

After multiplying (74) by 5 , we consider it modulo 13 :

$$
10 \cdot 16^{t}+2 \equiv-8(-1)^{k}+(-1)^{l}+(-1)^{m},
$$

i.e.

$$
16^{t+1}-2 \equiv 8(-1)^{k}+(-1)^{l+1}+(-1)^{m+1}(\bmod 13) .
$$

Taking into account that $16^{1} \equiv 3,16^{2} \equiv 9,16^{3} \equiv 1(\bmod 13)$, notice that the expression $8(-1)^{k}+(-1)^{l+1}+(-1)^{m+1}+2$ gives a residue of a power of 16 only in case when $k, l, m$ are odd; therefore $t \equiv 1(\bmod 3)$. Put $k=2 a+1$, $l=2 b+1, m=2 c+1, t=3 d+1, a, b, c, d \geq 0$. Then by (74),

$$
2^{12 d+5}+3=5^{4 a+2}+5^{4 b+1}+5^{4 c+1}, \quad a, b, c, d \geq 0 .
$$

Modulo 7 this gives $0 \equiv 2^{4 a+2}-2^{4 b+1}-2^{4 c+1}$, i.e., since $2^{4} \equiv 2(\bmod 7)$,

$$
2^{a+1} \equiv 2^{b}+2^{c}(\bmod 7)
$$

Since $2^{1} \equiv 2,2^{2} \equiv 4,2^{3} \equiv 1(\bmod 7)$, we evidently have three possibilities:

(a) $a \equiv b \equiv c \equiv 1(\bmod 3)$,

(b) $a \equiv b \equiv c \equiv 2(\bmod 3)$,

(c) $a \equiv b \equiv c \equiv 0(\bmod 3)$.

Let us consider each of them.

(a) $a \equiv b \equiv c \equiv 1(\bmod 3)$. Put $a=3 \beta+1, b=3 \gamma+1, c=3 \delta+1$, $\beta, \gamma, \delta \geq 0$. By (75) we have

$$
2^{12 d+5}+3=5^{12 \beta+6}+5^{12 \gamma+5}+5^{12 \delta+5}, \quad \beta, \gamma, \delta \geq 0 .
$$

Since $5^{6} \equiv-1(\bmod 601)$, we have $2^{12 d+5} \equiv-1+2 \cdot 5^{5}-3 \equiv 236(\bmod 601)$. However, the number 236 does not appear among the residues of powers of 2 modulo 601 (see above). We have a contradiction.

(b) $a \equiv b \equiv c \equiv 2(\bmod 3)$. Put $a=3 \beta+2, b=3 \gamma+2, c=3 \delta+2$, $\beta, \gamma, \delta \geq 0$. By (75) we have

$$
2^{12 d+5}+3=5^{12 \beta+10}+5^{12 \gamma+9}+5^{12 \delta+9}, \quad \beta, \gamma, \delta \geq 0 .
$$

By taking this modulo 601 we have $2^{12 d+5} \equiv 5^{10}+2 \cdot 5^{9}-3=-5^{4}-2 \cdot 5^{3}-3 \equiv$ $324(\bmod 601)$. We again obtain a contradiction.

(c) $a \equiv b \equiv c \equiv 0(\bmod 3)$. Put $a=3 \beta, b=3 \gamma, c=3 \delta, \beta, \gamma, \delta \geq 0$. By (75) we have

$$
2^{12 d+5}+3=5^{12 \beta+2}+5^{12 \gamma+1}+5^{12 \delta+1}, \quad \beta, \gamma, \delta \geq 0 .
$$


By taking this modulo 601 we have $2^{12 d+5} \equiv 25+10-3 \equiv 32(\bmod 601)$. Thus $12 d \equiv 0(\bmod 25)$, and so $d \equiv 0(\bmod 25)$. Put $d=25 \lambda, \lambda \geq 0$. By (76),

$$
2^{300 \lambda+5}+3=5^{12 \beta+2}+5^{12 \gamma+1}+5^{12 \delta+1}, \quad \lambda, \beta, \gamma, \delta \geq 0 .
$$

Now we show that $\gamma$ or $\delta$ is 0 . Indeed, if $\gamma, \delta \geq 1$, then $2^{300 \lambda+5} \equiv-3(\bmod 25)$. Consequently, $300 \lambda+5 \equiv 17(\bmod 20)$. This is a contradiction. By symmetry we can further suppose that $\delta=0$ in (76). We have

$$
2^{300 \lambda+5}-2=5^{12 \beta+2}+5^{12 \gamma+1}, \quad \lambda, \beta, \gamma \geq 0 .
$$

Now we show that also $\gamma=0$. Indeed, if $\gamma \geq 1$ then taking this modulo 25 gives

$$
2^{300 \lambda+5} \equiv 2(\bmod 25), \quad \text { so } 300 \lambda+5 \equiv 1(\bmod 20) .
$$

Again we obtain a contradiction.

Thus, $\gamma=0$ in (77). Therefore

$$
2^{300 \lambda+5}-7=5^{12 \beta+2} .
$$

If $\beta \geq 1$, then

$$
2^{300 \lambda+5} \equiv 7(\bmod 125) .
$$

Notice that $\min \left\{\alpha \geq 1: 2^{\alpha} \equiv 7(\bmod 125)\right\}=85$. Since $\varphi(125)=100$, we have $300 \lambda+5 \equiv 85(\bmod 100)$, and so $5 \equiv 85(\bmod 100)$. Again we have a contradiction. Therefore in (74), $\beta=0,2^{300 \lambda+5}=32$, and $\lambda=0$. Hence, we proved the following statement.

Proposition 13. The diophantine equation (72): $2^{\alpha}+3=5^{x}+5^{y}+5^{z}$ has the only solution: $\alpha=5, x=2, y=1, z=1$ in $x \geq y \geq z \geq 1, \alpha \geq 4$.

By a simple sorting out of the possibilities not included in Propositions 12, 13, using Proposition 8 we obtain

COROLlary 4. The diophantine equation $2^{\alpha}+3=5^{x}+5^{y}+5^{z}$ has only the following solutions (up to a permutation of $x, y, z$ ):

- $\alpha=7, x=3, y=1, z=0$;

- $\alpha=5, x=2, y=1, z=1$;

- $\alpha=3, x=1, y=1, z=0$;

- $\alpha=2, x=1, y=0, z=0$.

Now we are able to complete the proof of Theorem 2 when $q$ is a Fermat prime in the following more detailed form:

Proposition 14.

(i) If $q \geq 17$ is a Fermat prime, then $C_{2}^{!} \cap C_{q}^{!}=\{q+1, q+2,2 q, 2 q+1\}$.

(ii) $C_{2}^{!} \cap C_{3}^{!}=\{3,6,7,10,11,18,19\}$.

(iii) $C_{2}^{!} \cap C_{5}^{!}=\{6,7,10,11,35,130,131\}$. 
Proof. (i) According to Propositions 6 and 10 the solution $x=1, y=0$, $z=0, \alpha=\log _{2}(q-1)$ of (45) (or its permutation) corresponds to the elements $q+1, q+2$ of $C_{2}^{!} \cap C_{q}^{!}$, while the solution $x=1, y=1, z=0$, $\alpha=\log _{2}(q-1)$ (or its permutation) corresponds to the elements $2 q, 2 q+1$.

(ii) According to Proposition 6 and Corollary 3 the solution $\alpha=4$, $x=y=2, z=0$ of (45) (or its permutation) corresponds to the elements 18,19 of $C_{2}^{!} \cap C_{3}^{!}$; the solution $\alpha=2, x=y=1, z=0$ (or its permutations) corresponds to 6,7 , while the solution $\alpha=3, x=2, y=z=0$ (or its permutation) corresponds to 10,11. Finally, notice that by Proposition 1 the numbers $\leq 5$ are considered separately. Among them only $3 \in C_{2}^{!} \cap C_{3}^{!}$.

(iii) According to Proposition 6 and Corollary 4 the solution $\alpha=7$, $x=3, y=1, z=0$ (or its permutation) of (45) corresponds to the elements 130, 131 of $C_{2}^{!} \cap C_{5}^{!}$; the solution $\alpha=5, x=2, y=1, z=1$ (or its permutation) corresponds to 35 ; the solution $\alpha=3, x=1, y=1, z=0$ (or its permutation) corresponds to 10,11 , while the solution $\alpha=2, x=1$, $y=1, z=0$ (or its permutation) corresponds to 6,7 . Finally, $5 \notin C_{2}^{!} \cap C_{5}^{!}$. This completes the proof of Theorem 2.

5. Proof of Theorem 3. 1) Let $q$ be a prime in the interval $\left[2^{k-1}+5\right.$, $\left.2^{k}-1\right], q \neq\left(2^{k+2}+3\right) / 5$. By Theorem $2,\left|C_{2}^{!} \cap C_{q}^{!}\right|=2 t$ if and only if $2^{t-1} \leq(q-5) /\left(2^{k}-q\right)<2^{t}$, or

$$
\frac{2^{k+t-1}+5}{2^{t-1}+1} \leq q<\frac{2^{k+t}+5}{2^{t}+1}, \quad k \geq 3 .
$$

Put

Then

$$
\frac{2^{k+t-1}+5}{2^{t-1}+1}=x, \quad \frac{2^{k+t}+5}{2^{t}+1}=x+\triangle x
$$

$$
\triangle x=\frac{2^{k+t-1}-5 \cdot 2^{t-1}}{\left(2^{t-1}+1\right)\left(2^{t}+1\right)} \sim \frac{1}{2^{t}+1} x \quad(k \rightarrow \infty) .
$$

By the prime number theorem, for $\lambda=1 /\left(2^{t}+1\right)$,

$$
\pi(x+\triangle x)-\pi(x) \sim \frac{\lambda x}{\log x} \quad(x \rightarrow \infty) .
$$

Therefore, the number of primes $q$ in $\left[2^{k-1}+5,2^{k}-1\right]$ for which $\left|C_{2}^{!} \cap C_{q}^{!}\right|=2 t$ is

$$
\begin{aligned}
\frac{\lambda x}{\pi\left(2^{k-1}\right) \log x} & \sim \frac{2^{k+t-1}}{\left(2^{t}+1\right)\left(2^{t-1}+1\right)} \cdot \frac{k-1}{2^{k-1}(k+t-1)} \\
& \sim \frac{2^{t}}{\left(2^{t-1}+1\right)\left(2^{t}+1\right)} \quad(k \rightarrow \infty),
\end{aligned}
$$

whence the first formula of Theorem 3 evidently follows. 
REMARK 5. In particular, we see that

$$
\sum_{t=1}^{\infty} \frac{2^{t}}{\left(2^{t-1}+1\right)\left(2^{t}+1\right)}=1
$$

It also follows directly that

$$
\begin{aligned}
\sum_{t=1}^{\infty} \frac{2^{t}}{\left(2^{t-1}+1\right)\left(2^{t}+1\right)} & =2 \sum_{t=1}^{\infty}\left(\frac{1}{2^{t-1}+1}-\frac{1}{2^{t}+1}\right) \\
& =2\left(\frac{1}{2}-\frac{1}{3}+\frac{1}{3}-\frac{1}{5}+\frac{1}{5}-\cdots\right) .
\end{aligned}
$$

2) Further, by (78) the lim sup on the left-hand side of the second formula of Theorem 3 is attained for the sequence

$$
n_{m}^{\prime}=\left\lfloor\frac{2^{m+t}+5}{2^{t}+1}\right\rfloor,
$$

while the liminf of the same expression is attained for the sequence

$$
n_{m}^{\prime \prime}=\left\lfloor\frac{2^{m-1+t}+5}{2^{t-1}+1}\right\rfloor .
$$

Thus we have

$$
\begin{aligned}
& \limsup _{n \rightarrow \infty} \frac{\left|\left\{q \in P, q \leq n:\left|C_{2}^{!} \cap C_{q}^{!}\right|=2 t\right\}\right|}{\pi(n)} \\
& =\lim _{m \rightarrow \infty} \frac{\sum_{k \leq m}\left(\pi\left(\frac{2^{k+t}+5}{2^{t}+1}\right)-\pi\left(\frac{2^{k+t-1}+5}{2^{t-1}+1}\right)\right)}{\pi\left(\frac{2^{m+t}+5}{2^{t}+1}\right)} \\
& =\lim _{m \rightarrow \infty} \frac{\sum_{k \leq m}\left(\pi\left(\frac{2^{k+t}}{2^{t}+1}\right)-\pi\left(\frac{2^{k+t}}{2^{t}+2}\right)\right)}{\pi\left(\frac{2^{m+t}}{2^{t}+1}\right)} \\
& =\lim _{m \rightarrow \infty} \frac{\sum_{k \leq m} \pi\left(\frac{2^{k+t}}{\left(2^{t}+1\right)\left(2^{t}+2\right)}\right)}{\pi\left(\frac{2^{m+t}}{2^{t}+1}\right)}=\frac{1}{2^{t-1}+1} \lim _{m \rightarrow \infty} \frac{\sum_{k \leq m} \pi\left(2^{k+t-1}\right)}{\pi\left(2^{m+t}\right)} .
\end{aligned}
$$

Since by the Bertrand postulate $\pi\left(2^{m+t+1}\right)>\pi\left(2^{m+t}\right)$, the classical Stolz theorem and the prime number theorem yield

$$
\lim _{m \rightarrow \infty} \frac{\sum_{k \leq m} \pi\left(2^{k+t-1}\right)}{\pi\left(2^{m+t}\right)}=\lim _{m \rightarrow \infty} \frac{\pi\left(2^{m+t-1}\right)}{\pi\left(2^{m+t}\right)-\pi\left(2^{m+t-1}\right)}=1 .
$$

Thus the second formula of Theorem 3 follows. 
Analogously,

$$
\begin{aligned}
& \liminf _{n \rightarrow \infty} \frac{\mid\{q \in P, q \leq n}{} \frac{\left.\left|C_{2}^{!} \cap C_{q}^{!}\right|=2 t\right\} \mid}{\pi(n)} \\
&=\lim _{m \rightarrow \infty} \frac{\sum_{k \leq m-1}\left(\pi\left(\frac{2^{k+t}+5}{2^{t}+1}\right)-\pi\left(\frac{2^{k+t-1}+5}{2^{t-1}+1}\right)\right)}{\pi\left(\frac{2^{m-1+t}+5}{2^{t-1}+1}\right)} \\
&=\lim _{m \rightarrow \infty} \frac{\sum_{k \leq m-1} \pi\left(\frac{2^{k+t}}{\left(2^{t}+1\right)\left(2^{t}+2\right)}\right)}{\pi\left(\frac{2^{m+t-1}}{2^{t-1}+1}\right)} \\
&=\frac{1}{2^{t}+1} \lim _{m \rightarrow \infty} \frac{\sum_{k \leq m-1} \pi\left(2^{k+t-1}\right)}{\pi\left(2^{m+t-1}\right)}=\frac{1}{2^{t}+1} .
\end{aligned}
$$

6. Some corollaries. Since the function $(x-5) /\left(2^{k}-x\right)$ increases for any fixed $k \geq 4$, from (44) we obtain the following result.

Corollary 5. If $q$ is a prime $\geq 7$, then in the estimate

$$
\left|C_{2}^{!} \cap C_{q}^{!}\right| \leq 2\left(1+\left\lfloor\log _{2}(q-5)\right\rfloor\right)
$$

we have equality if and only if $q$ is a Mersenne prime.

Further, from (79) and Proposition 2 we have:

Corollary 6. If $7 \leq q<2^{k}$, then

$$
2 \leq\left|C_{2}^{!} \cap C_{q}^{!}\right| \leq 2 k \text {. }
$$

Moreover, the upper bound in (80) is attained if and only if $q$ is a Mersenne prime.

Corollary 7. If $q \geq 7$ and $n \in C_{2}^{!} \cap C_{q}^{!}$, then $n \leq \frac{1}{2}(q+1)^{2}+3$. Equality holds for Mersenne primes $\geq 31$.

Proof. From the proof of Theorem 2 it follows that

$$
\begin{aligned}
S_{q}: & =\max \left\{n: n \in C_{2}^{!} \cap C_{q}^{!}\right\} \\
& = \begin{cases}19, & q=3, \\
131, & q=5, \\
5 q-1, & q=\left(2^{4 k+1}+3\right) / 5, k \geq 1, \\
2 q+1, & q=2^{2^{k-1}}+1, k \geq 3, \\
2 q-3, & q=2^{k}+3, k \geq 3, \\
2^{k+\left\lfloor\log _{2} \frac{q-5}{2^{k}-q}\right\rfloor}+3, & 2^{k-1}+5 \leq q \leq 2^{k}-1, \\
& k \geq 4, q \neq\left(2^{k+2}+3\right) / 5 .\end{cases}
\end{aligned}
$$

Therefore, for $q \geq 31$ the maximal value of $S_{q}$ is attained at a Mersenne 
prime $q=2^{k}-1, k \geq 5$. Thus, for $q \geq 31$,

$$
S_{q} \leq(q+1) 2^{\left\lfloor\log _{2}(q-5)\right\rfloor}+3=(q+1) \frac{q+1}{2}+3=\frac{1}{2}(q+1)^{2}+3,
$$

and this estimate, according to (81), is true for $q \geq 7$.

COROllary 8. For $q=7$ and all non-Fermat primes $q \geq 13, q \in$ $\left(2^{k-1}, 2^{k}\right), k \geq 4$, that do not have the forms $2^{n}+3,\left(2^{n}+3\right) / 5(n>3)$ and for which

$$
\left\lfloor\log _{2} \frac{q-1}{2^{k}-q}\right\rfloor \nmid k
$$

we have

$$
\left|C_{2}^{!} \cap C_{q}^{!}\right|=2 \max \left\{\alpha \in \mathbb{N}: \exists m,\left\lfloor 2^{m} / q\right\rfloor=2^{\alpha-1}\right\} .
$$

Proof. From the equality $\left\lfloor 2^{m} / q\right\rfloor=2^{\alpha-1}$, we find

$$
2^{m-\alpha} \leq \frac{2^{m}}{2^{\alpha-1}+1}<q<2^{m-\alpha+1} .
$$

So, $m-\alpha+1=k$. Therefore, by (84),

$$
2^{m-\alpha+1}-\frac{2^{m}}{2^{\alpha-1}+1}>2^{k}-q,
$$

whence $2^{k} /\left(2^{k}-q\right)>2^{\alpha-1}+1,2^{\alpha-1}<q /\left(2^{k}-q\right)$. Thus,

$$
\max \alpha=1+\left\lfloor\log _{2} \frac{q}{2^{k}-q}\right\rfloor .
$$

By Theorem 2 it is left to prove that

$$
\left\lfloor\log _{2} \frac{q}{2^{k}-q}\right\rfloor=\left\lfloor\log _{2} \frac{q-5}{2^{k}-q}\right\rfloor .
$$

If (85) is not true, then there exists $\beta \in \mathbb{N}$ so that

$$
\frac{q-5}{2^{k}-q}<2^{\beta} \leq \frac{q}{2^{k}-q},
$$

so that

$$
2^{k+\beta} \leq q\left(2^{\beta}+1\right)<2^{k+\beta}+5 .
$$

But if $q\left(2^{\beta}+1\right)=2^{k+\beta}+3$, then by Lemma $7, q$ has the form $\left(2^{n}+3\right) / 5$, contrary to assumption. Thus by (86),

$$
q\left(2^{\beta}+1\right)=2^{k+\beta}+1,
$$

whence $\beta \mid k$ and $\beta=\left\lfloor\log _{2} \frac{q-1}{2^{k}-q}\right\rfloor$. The latter contradicts (82). In addition notice that formula (83) is also true for $q=7$.

Corollary 9. For every $t \geq 1$ the set of primes

$$
\left\{q \in P:\left|C_{2}^{!} \cap C_{q}^{!}\right|=2 t\right\}
$$


is infinite. Moreover, every arithmetic progression $a, a+d, a+2 d, \ldots$ with $(a, d)=1$ contains infinitely many primes of this type for every fixed $t$.

Proof. If, for a fixed $t, x_{k}=\left(2^{k+t-1}+5\right) /\left(2^{t-1}+1\right)$, then the interval (78) contains only primes under consideration and has length asymptotically equal to $x_{k} /\left(2^{t}+1\right)(k \rightarrow \infty)$. Consequently, as is well known [10], it contains asymptotically $x_{k} / \varphi(d)\left(2^{t}+1\right) \log x_{k}$ primes belonging to the above arithmetic progression.

7. Proof of Theorem 4. In the theory of the existence of primes in short intervals the best result known to date is due to Baker, Harman and Pintz [2]. They showed for sufficiently large $x$ the existence of a prime in the interval $\left(x, x+x^{0.525}\right)$ and, moreover, obtained the estimate

$$
\pi\left(x+x^{0.525}\right)-\pi(x)>0.09 \frac{x^{0.525}}{\log x} .
$$

According to Theorem 2 the interval (78) contains only primes $q \in\left(2^{k-1}+3\right.$, $\left.2^{k}-1\right), k \geq 4$, for which $\left|C_{2}^{!} \cap C_{q}^{!}\right|=2 t$ if $q \neq\left(2^{k+2}+3\right) / 5$.

We shall show that for each $t \in[1,0.475 k-1](k \geq 5)$ the interval (78) contains an interval of type $\left[x, x+x^{0.525}\right]$. Indeed, we have

$$
\begin{aligned}
\frac{2^{k+t}+5}{2^{t}+1} & =\frac{2^{k+t-1}+5}{2^{t-1}+1}+\frac{2^{k+t-1}-5 \cdot 2^{t-1}}{\left(2^{t-1}+1\right)\left(2^{t}+1\right)} \\
& >\frac{2^{k+t-1}+5}{2^{t-1}+1}+2^{k-t-1} \geq \frac{2^{k+t-1}+5}{2^{t-1}+1}+2^{0.525 k} \\
& =\frac{2^{k+t-1}+5}{2^{t-1}+1}+\left(\frac{2^{k+t-1}+2^{k}}{2^{t-1}+1}\right)^{0.525} \\
& \geq \frac{2^{k+t-1}+5}{2^{t-1}+1}+\left(\frac{2^{k+t-1}+5}{2^{t-1}+1}\right)^{0.525} .
\end{aligned}
$$

By (87) for sufficiently large $k$ the number of primes for which $\left|C_{2}^{!} \cap C_{q}^{!}\right|=2 t$ with $t \in[1,0.475 k-1]$ is not less than

$$
0.09\left(\frac{2^{k+t-1}+5}{2^{t-1}+1}\right)^{0.525} / \ln \frac{2^{k+t-1}+2^{k}}{2^{t-1}+1} \geq \frac{0.09}{\log 2} \cdot \frac{2^{0.525(k-1)}}{k} .
$$

Remark 6. Notice that if H. Cramer's 1937 conjecture ([8, A2])

$$
\limsup _{n \rightarrow \infty} \frac{p_{n+1}-p_{n}}{\left(\ln p_{n}\right)^{2}}=1
$$

where $p_{n}$ is the $n$th prime, is true, then in Theorem 4(i) for large enough $k$ the number $\left|C_{2}^{!} \cap C_{q}^{!}\right|$assumes all even values in the interval $[2,\lfloor(1-\varepsilon) k\rfloor]$. 
8. Proof of Theorem 5. Notice that from Theorem 2 it follows that

$$
\begin{array}{lll}
q(1)=37, & q(2)=13, & \\
q(4)=29, & q(5)=31, & \ldots
\end{array}
$$

Let $t \geq 5$. For $k \geq t$ consider the number

$$
\varrho=\varrho(k, t)=\min \left\{j \geq 2^{k-t}: 3 \leq 2^{k}-j \in P\right\} .
$$

It is evident that

$$
2^{k-t} \leq \varrho, \quad \text { i.e. } \quad k-t \leq\left\lfloor\log _{2} \varrho\right\rfloor .
$$

Lemma 10. If

$$
q=2^{k}-\varrho \in P,
$$

then in (89) equality holds if and only if

$$
\left|C_{2}^{!} \cap C_{q}^{!}\right|=2 t, \quad t \geq 5 .
$$

Proof. It is evident that $q>2^{k-1}$ and $\varrho<2^{k-1}$. Let (91) be valid. Then by Theorem 2 for the prime $q$ of (90) we have

$$
\begin{aligned}
t & =1+\left\lfloor\log _{2} \frac{q-5}{2^{k}-q}\right\rfloor=1+\left\lfloor\log _{2} \frac{2^{k}-\varrho-5}{\varrho}\right\rfloor \\
& \leq 1+\left\lfloor\log _{2}\left(2^{k}-\varrho-5\right)\right\rfloor-\left\lfloor\log _{2} \varrho\right\rfloor \leq 1+k-1-\left\lfloor\log _{2} \varrho\right\rfloor \\
& =k-\left\lfloor\log _{2} \varrho\right\rfloor
\end{aligned}
$$

i.e. $k-t \geq\left\lfloor\log _{2} \varrho\right\rfloor$. Therefore, in (89) we have equality.

Conversely, let

$$
k-t=\left\lfloor\log _{2} \varrho\right\rfloor .
$$

Then by Theorem 2 and (90) we have

$$
\begin{aligned}
\left|C_{2}^{!} \cap C_{q}^{!}\right| & =2\left(1+\left\lfloor\log _{2} \frac{q-5}{2^{k}-q}\right\rfloor\right)=2\left(1+\left\lfloor\log _{2} \frac{2^{k}-\varrho-5}{\varrho}\right\rfloor\right) \\
& \leq 2\left(1+\left\lfloor\log _{2}\left(2^{t+\left\lfloor\log _{2} \varrho\right\rfloor}-\varrho-5\right)\right\rfloor-\left\lfloor\log _{2} \varrho\right\rfloor\right) \\
& =2\left(1+t+\left\lfloor\log _{2} \varrho\right\rfloor-1-\left\lfloor\log _{2} \varrho\right\rfloor\right)=2 t .
\end{aligned}
$$

On the other hand,

$$
\begin{aligned}
\left|C_{2}^{!} \cap C_{q}^{!}\right| & =2\left(1+\left\lfloor\log _{2} \frac{2^{k}-\varrho-5}{\varrho}\right\rfloor\right) \\
& \geq 2\left(1+\left\lfloor\left\lfloor\log _{2}\left(2^{k}-\varrho-5\right)\right\rfloor-\log _{2} \varrho\right\rfloor\right) \\
& =2\left(1+\left\lfloor\log _{2}\left(2^{k}-\varrho-5\right)\right\rfloor-\left\lfloor\log _{2} \varrho\right\rfloor\right) \\
& =2(1+k-1-k+t)=2 t .
\end{aligned}
$$

So, $\left|C_{2}^{!} \cap C_{q}^{!}\right|=2 t$. 
Corollary 10. Let

$$
k_{0}(t)=\min \left\{k \geq t: k-t=\left\lfloor\log _{2} \varrho(t, k)\right\rfloor\right\} .
$$

Then

$$
q(t)=2^{k_{0}(t)}-\varrho .
$$

Now we complete the proof of Theorem 5 . Put $x=2^{k}-2^{0.525 k}$. Then $x+x^{0.525}=2^{k}-2^{0.525 k}+2^{0.525 k}\left(1-\frac{1}{2^{0.475 k}}\right)^{0.525}=2^{k}-\frac{0.525}{2^{0.475 k}}-\cdots<2^{k}$.

So by (88)-(89), taking into account the above result of Baker, Harman and Pintz, for sufficiently large $k$, say $k \geq k_{1}$, we have

$$
2^{k-t} \leq \varrho \leq 2^{\max (0.525 k, k-t)} .
$$

Therefore, the condition $k-t=\left\lfloor\log _{2} \varrho(t, k)\right\rfloor$ in (93) is satisfied at least for $k \geq \max \left(t / 0.475, k_{1}\right)$. Consequently, for large enough $t$, namely $t \geq 0.475 k_{1}$, we have $k_{0} \leq\lceil 40 t / 19\rceil$. By (94) we conclude that $q(t) \leq 2^{\lceil 40 t / 19\rceil}$.

REMARK 7. According to Cramer's above-mentioned conjecture the interval $\left(x, x+x^{\varepsilon}\right)$ must contain a prime for sufficiently large $x$, namely $x \geq x_{\varepsilon}$. In this case in the same way one can prove that for $t \geq(1-\varepsilon) x_{\varepsilon}$ we have $q(t)=2^{\lceil t /(1-\varepsilon)\rceil}$.

9. Numerical results. 1) Theorem 2 and Proposition 3 give a possibility to fill the following tables, except for the cases $3 \leq p<q \leq 47$ for which $(q-1) /(p-1)$ is a power of 2 .

Table 1. The cardinality of $C_{p}^{!} \cap C_{q}^{!}$for $2 \leq p<q \leq 47$

\begin{tabular}{c|cccccccccccccc}
\hline$q \backslash p$ & 2 & 3 & 5 & 7 & 11 & 13 & 17 & 19 & 23 & 29 & 31 & 37 & 41 & 43 \\
\hline 3 & 7 & & & & & & & & & & & & & \\
5 & 7 & $?$ & & & & & & & & & & & & \\
7 & 6 & 8 & 9 & & & & & & & & & & & \\
11 & 3 & 4 & 9 & 22 & & & & & & & & & & \\
13 & 4 & 3 & 11 & $?$ & 23 & & & & & & & & & \\
17 & 4 & $?$ & $?$ & 13 & 23 & 22 & & & & & & & & \\
19 & 3 & 2 & 10 & 16 & 38 & 25 & 42 & & & & & & & \\
23 & 4 & 0 & 7 & 28 & 28 & 53 & 29 & 37 & & & & & & \\
29 & 8 & 3 & 16 & 13 & 15 & 24 & 48 & 39 & 40 & & & & & \\
31 & 10 & 6 & 25 & 11 & 18 & 21 & 61 & 46 & 39 & 90 & & & & \\
37 & 2 & 3 & 8 & 14 & 36 & 22 & 36 & $?$ & 52 & 50 & 63 & & & \\
41 & 2 & 3 & 5 & 21 & $?$ & 36 & 27 & 42 & 73 & 53 & 52 & 96 & & \\
43 & 2 & 3 & 5 & 21 & 26 & 39 & 25 & 33 & 109 & 57 & 55 & 81 & 147 & \\
47 & 4 & 3 & 10 & 32 & 19 & 69 & 26 & 29 & 79 & 68 & 63 & 64 & 93 & 120 \\
\hline
\end{tabular}


Notice that for the exceptional cases the count up to $10^{8}$ shows that $\left|C_{3}^{!} \cap C_{5}^{!}\right| \geq 25, \quad\left|C_{3}^{!} \cap C_{17}^{!}\right| \geq 10, \quad\left|C_{5}^{!} \cap C_{17}^{!}\right| \geq 23, \quad\left|C_{7}^{!} \cap C_{13}^{!}\right| \geq 66$,
$\left|C_{11}^{!} \cap C_{41}^{!}\right| \geq 76, \quad\left|C_{19}^{!} \cap C_{37}^{!}\right| \geq 175$.

Table 2. The maximal elements $M_{p, q}$ of the sets $C_{p}^{!} \cap C_{q}^{!}, 2 \leq p<q \leq 47$

\begin{tabular}{c|cccccccccccccc}
\hline$q \backslash p$ & 2 & 3 & 5 & 7 & 11 & 13 & 17 & 19 & 23 & 29 & 31 & 37 & 41 & 43 \\
\hline 3 & 19 & & & & & & & & & & & & & \\
5 & 131 & 2 & & & & & & & & & & & & \\
7 & 34 & 20 & 20 & & & & & & & & & & & \\
11 & 19 & 20 & 24 & 98 & & & & & & & & & & \\
13 & 35 & 20 & 38 & $?$ & 54 & & & & & & & & & \\
17 & 35 & $?$ & $?$ & 50 & 50 & 38 & & & & & & & & \\
19 & 35 & 20 & 39 & 55 & 170 & 56 & 152 & & & & & & & \\
23 & 67 & - & 39 & 202 & 98 & 390 & 68 & 94 & & & & & & \\
29 & 259 & 71 & 260 & 55 & 54 & 116 & 144 & 86 & 68 & & & & & \\
31 & 575 & 518 & 524 & 55 & 92 & 64 & 278 & 154 & 92 & 260 & & & & \\
37 & 67 & 71 & 74 & 104 & 332 & 110 & 152 & $?$ & 184 & 86 & 154 & & & \\
41 & 67 & 71 & 74 & 202 & $?$ & 204 & 84 & 170 & 368 & 122 & 92 & 332 & & \\
43 & 67 & 71 & 74 & 202 & 175 & 207 & 84 & 94 & 730 & 128 & 128 & 184 & 696 & \\
47 & 131 & 71 & 134 & 1550 & 98 & 1550 & 140 & 94 & 390 & 234 & 140 & 110 & 204 & 386 \\
\hline
\end{tabular}

Notice that for the exceptional cases the count until $10^{8}$ shows that

$$
\begin{array}{rll}
M_{3,5} \geq 524306, & M_{3,17} \geq 262160, & M_{5,17} \geq 262164, \\
M_{7,13} \geq 25165860, & M_{11,41} \geq 20503, & M_{19,37} \geq 18874439 .
\end{array}
$$

Further, notice that Proposition 4 gives a qualitative explanation of the variation of the numbers $M_{p, q}$, in particular, near the "resonance points" $(p, q)$, for which $(q-1) /(p-1)$ is a power of 2 .

2) The list of primes $\leq 10^{8}$ for which formula (83) of Corollary 8 is not true contains only 25 primes. They are:

$3,5,11,13,17,19,43,67,103,131,241,257,683,2731,4099,32771,43691$,

61681, 65537, 65539, 174763, 262147, 2796203, 6710887, 15790321.

3) Evaluation of the function $q(t)$. Notice that formulas (88), (93), (94) give a simple algorithm for finding the values of $q(t)$. It follows from Theorem 5 that the running time of this algorithm is $O(t)$ with the implicit constant in $O(\ldots)$ not exceeding $40 / 19$.

EXAmple. Let $t=6$. If $k=6$, then $\varrho=64-61=3$, but $6 \neq 6-\left\lfloor\log _{2} 3\right\rfloor$; if $k=7$, then $\varrho=128-113=15$, but $6 \neq 7-\left\lfloor\log _{2} 15\right\rfloor$; if $k=8$, then 
$\varrho=256-251=5$ and $6=8-\left\lfloor\log _{2} 5\right\rfloor$. Therefore, by (93), (94), $k_{0}=8$, $\varrho=5$, and $q(6)=2^{8}-5=251$.

Table 3. Values of $q(t)$

\begin{tabular}{ccccccccccc}
\hline$t$ & 1 & 2 & 3 & 4 & 5 & 6 & 7 & 8 & 9 & 10 \\
$q(t)$ & $2^{6}-27$ & $2^{4}-3$ & $2^{3}-3$ & $2^{5}-3$ & $2^{5}-1$ & $2^{8}-5$ & $2^{7}-1$ & $2^{9}-3$ & $2^{10}-3$ & $2^{12}-5$ \\
\hline$t$ & 11 & 12 & 13 & 14 & 15 & 16 & 17 & 18 & 19 \\
$q(t)$ & $2^{12}-3$ & $2^{16}-17$ & $2^{13}-1$ & $2^{17}-9$ & $2^{18}-11$ & $2^{18}-5$ & $2^{17}-1$ & $2^{20}-5$ & $2^{19}-1$ \\
\hline$t$ & 20 & 21 & 22 & 23 & 24 & 25 & 26 & 27 & 28 \\
$q(t)$ & $2^{23}-15$ & $2^{22}-3$ & $2^{26}-27$ & $2^{24}-3$ & $2^{26}-3$ & $2^{30}-35$ & $2^{31}-61$ & $2^{31}-19$ & $2^{29}-3$ \\
\hline
\end{tabular}

4) On numbers $n$ for which $e_{p}(n)$ and $e_{q}(n)$ are powers of an odd prime $\nu$. The following two tables are based on a natural generalization of Proposition 3. Let $\nu \geq 2$ be a prime. For a prime $p$ define $C_{\nu, p}^{!}=\{n \in \mathbb{N}: \exists \alpha \in$ $\left.\mathbb{Z}_{+}, e_{p}(n)=\nu^{\alpha}\right\}$. One can prove, similarly to Proposition 3 , the following generalization.

Proposition 15. If $p<q$ are primes so that $(q-1) /(p-1)$ is not a power of $\nu$, then the set $C_{\nu, p}^{!} \cap C_{\nu, q}^{!}$is finite. Moreover, if $n \in C_{\nu, p}^{!} \cap C_{\nu, q}^{!}$, then

$$
n \in\left[q, 2\left(1+\frac{\log 2}{2 \log q-1}\right) q^{2}-1\right) .
$$

Notice that if $\nu \geq 3$ and $p=2$ then $(q-1) /(p-1)$ is never a power of $\nu$ and thus all sets $C_{\nu, 2}^{!} \cap C_{\nu, q}^{!}$are finite. In addition consider the case $p=2, q=\nu$.

Proposition 16. For $\nu \geq 5$ we have $\left|C_{\nu, 2}^{!} \cap C_{\nu, \nu}^{!}\right|=0$ or 2.

Proof. Let $n \in C_{\nu, 2}^{!} \cap C_{\nu, \nu}^{!}$. By Proposition 16 for $q=\nu \geq 5$ we have

$$
n \leq 2\left(1+\frac{\log 2}{2 \log 5-1}\right) \nu^{2}-1=2.62 \ldots \nu^{2}-1 .
$$

Therefore, $e_{\nu}(n)=1$ or $\nu$. Notice now that $n \notin\left[2 \nu, \nu^{2}-1\right]$ (else $2 \leq e_{\nu}(n) \leq$ $\nu-1)$ and $n \notin\left[\nu^{2}, 2.62 \ldots \nu^{2}-1\right]$ (else $\left.e_{\nu}(n) \geq \nu+1\right)$. Thus, $n \leq 2 \nu-1$ and $e_{\nu}(n)=1$. Further, $n \geq \nu \geq 5$, therefore $e_{2}(n) \geq 3$, and consequently $e_{2}(n) \geq \nu$. Moreover, $e_{2}(n)<n \leq 2 \nu-1$. Thus, $e_{2}(n)=\nu$, and if $n$ is even, also $n+1 \in C_{\nu, 2}^{!} \cap C_{\nu, \nu}^{!}$. Since $e_{2}(n-2) \leq \nu-1$ and $e_{2}(n+2) \geq \nu+1$, we have $\left|C_{\nu, 2}^{!} \cap C_{\nu, \nu}^{!}\right|=2$ or 0 .

In addition, notice that if an even $n \in C_{\nu, 2}^{!} \cap C_{\nu, \nu}^{!}$is given then by Lemmas 3, 4 using the facts that $n \leq 2 \nu-2, e_{2}(n)=\nu$ we have

$$
\begin{aligned}
\nu+1 & \leq n=e_{2}(n)+\sigma_{2}(n) \leq \nu+\log _{2}(n+1) \\
& \leq \nu+\log _{2}(2 \nu-1)<\nu+1+\log _{2} \nu .
\end{aligned}
$$


Thus, if for some even $n \in\left[\nu+1, \nu+1+\log _{2} \nu\right)$ we have $e_{2}(n-2) \leq \nu-1$ and $e_{2}(n) \geq \nu+1$, then

$$
C_{\nu, 2}^{!} \cap C_{\nu, \nu}^{!}=\emptyset .
$$

According to Proposition 16 we have a partition of the set of all primes $\geq 5$ into two subsets. In Table 4 we provide the value of $\left|C_{\nu, 2}^{!} \cap C_{\nu, \nu}^{!}\right|$for $\nu<200$.

Table 4. The numbers $\lambda_{\nu}=\left|C_{\nu, 2}^{!} \cap C_{\nu, \nu}^{!}\right|, 5 \leq \nu \leq 199$

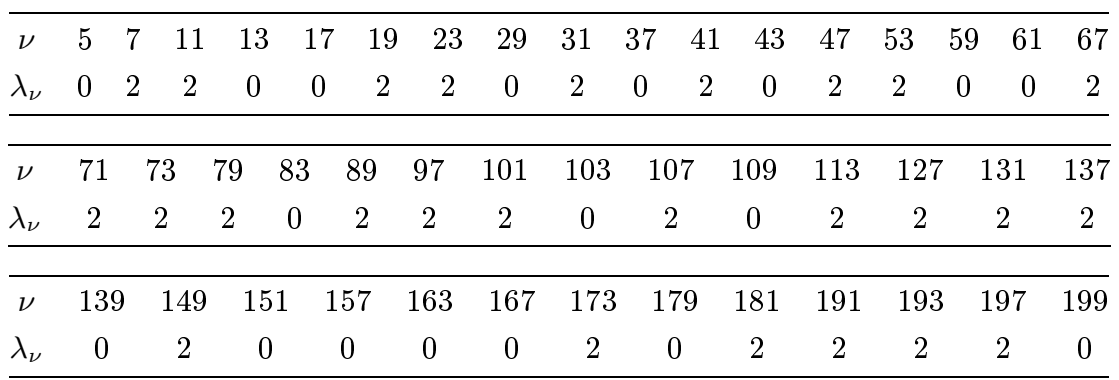

Table 5. The cardinality of $C_{3, p}^{!} \cap C_{3, q}^{!}$for $2 \leq p<q \leq 61$

\begin{tabular}{c|ccccccccccccccccc}
\hline$q \backslash p$ & 2 & 3 & 5 & 7 & 11 & 13 & 17 & 19 & 23 & 29 & 31 & 37 & 41 & 43 & 47 & 53 & 59 \\
\hline 3 & 3 & & & & & & & & & & & & & & & & \\
5 & 1 & 1 & & & & & & & & & & & & & & \\
7 & 0 & $?$ & 3 & & & & & & & & & & & & & & \\
11 & 0 & 1 & 9 & 4 & & & & & & & & & & & & & \\
13 & 0 & 3 & $?$ & 6 & 14 & & & & & & & & & & & & \\
17 & 0 & 9 & 3 & 16 & 6 & 10 & & & & & & & & & & & \\
19 & 0 & 2 & 1 & $?$ & 8 & 7 & 26 & & & & & & & & & & \\
23 & 2 & 1 & 5 & 5 & 11 & 10 & 11 & 22 & & & & & & & & & \\
29 & 0 & 1 & 6 & 2 & 33 & 13 & 12 & 10 & 22 & & & & & & & & \\
31 & 0 & 3 & 10 & 6 & $?$ & 20 & 14 & 12 & 15 & 61 & & & & & & & \\
37 & 0 & 3 & $?$ & 7 & 7 & $?$ & 17 & 18 & 14 & 26 & 38 & & & & & & \\
41 & 0 & 3 & 4 & 7 & 3 & 18 & 28 & 19 & 18 & 17 & 22 & 59 & & & & & \\
43 & 2 & 6 & 2 & 11 & 1 & 10 & 34 & 20 & 20 & 15 & 19 & 50 & 97 & & & & \\
47 & 2 & 6 & 0 & 14 & 0 & 5 & 58 & 36 & 23 & 18 & 16 & 34 & 58 & 77 & & & \\
53 & 2 & 12 & 0 & 21 & 7 & 0 & 26 & 76 & 28 & 24 & 22 & 21 & 34 & 46 & 70 & & \\
59 & 2 & 1 & 3 & 4 & 11 & 1 & 9 & 30 & 46 & 29 & 28 & 22 & 23 & 27 & 46 & 82 & \\
61 & 2 & 0 & 5 & 2 & 11 & 5 & 7 & 22 & 58 & 29 & 30 & 24 & 21 & 25 & 38 & 74 & 156 \\
\hline
\end{tabular}

Notice that for the exceptional cases for which $(q-1) /(p-1)$ is a power of 3 , the count up to $10^{8}$ shows that 


$$
\begin{gathered}
\left|C_{3,3}^{!} \cap C_{3,7}^{!}\right| \geq 8, \quad\left|C_{3,5}^{!} \cap C_{3,13}^{!}\right| \geq 18, \quad\left|C_{3,3}^{!} \cap C_{3,19}^{!}\right| \geq 7, \quad\left|C_{3,7}^{!} \cap C_{3,19}^{!}\right| \geq 29, \\
\left|C_{3,11}^{!} \cap C_{3,31}^{!}\right| \geq 65, \quad\left|C_{3,5}^{!} \cap C_{3,37}^{!}\right| \geq 17, \quad\left|C_{3,13}^{!} \cap C_{3,37}^{!}\right| \geq 75 .
\end{gathered}
$$

REMARK 8. One can prove, similarly to Proposition 4, the following generalization that gives a qualitative explanation of the variation of the maximal elements $M_{\nu, p, q}$ of the sets $C_{\nu, p}^{!} \cap C_{\nu, q}^{!}$.

Proposition 17. If $\nu$ is an odd prime and $p<q$ are primes so that $(q-1) /(p-1)$ is not a power of $\nu$, then for $n \in C_{\nu, p}^{!} \cap C_{\nu, q}^{!}$we have

$$
\frac{n}{\log (n+1)} \leq \max \left(a_{\nu}(p, q), b_{\nu}(p, q)\right)
$$

where

$$
\begin{aligned}
& a_{\nu}(p, q)=\frac{p-1}{\log p}\left(1-\nu^{-\left\{\log _{\nu} \frac{q-1}{p-1}\right\}}\right)^{-1}, \\
& b_{\nu}(p, q)=\frac{q-1}{\log q}\left(1-\nu^{\left\{\log _{\nu} \frac{q-1}{p-1}\right\}^{-1}}\right)^{-1} .
\end{aligned}
$$

\section{Open problems}

1. Is $C_{p}^{!}$infinite for $p \geq 3$ ?

Due to the fact that $C_{p}$ is of density close to 1 by Theorem 1 , we expect the answer to be in the affirmative.

2. Is the set $C_{p}^{!} \cap C_{q}^{!}$finite for primes $3 \leq p<q$ with $(q-1) /(p-1)$ a power of 2 ?

3. Does the diophantine equation $\sigma_{p}(n)=\sigma_{q}(n)$, where $p \neq q$ are fixed primes, have infinitely many solutions?

4. Is the set of primes $q$ for which $\left|C_{2}^{!} \cap C_{q}^{!}\right|=3$ infinite?

Notice that by Theorem 2 this question is equivalent to the question about the infinity of primes of the form $2^{n}+3$.

5. Is the set of primes $q$ for which $\left|C_{2}^{!} \cap C_{q}^{!}\right|=5$ infinite?

By Theorem 2, this question is equivalent to the question about the infinity of primes of the form $\left(2^{n}+3\right) / 5$.

6. Is the set of primes $q$ for which $\left|C_{2}^{!} \cap C_{q}^{!}\right| \neq 2 \max \left\{\alpha \in \mathbb{N}: \exists m,\left\lfloor 2^{m} / q\right\rfloor\right.$ $\left.=2^{\alpha-1}\right\}$, infinite?

The question arises in view of Corollary 8 .

7. Find a generalization of Theorem 2 to the set $C_{\nu, \nu}^{!} \cap C_{\nu, q}^{!}, \nu<q$ (see Sections 7, 4).

Remark 9. Together with Proposition 15, one can obtain a generalization of Proposition 5: if $p<q$ are primes so that $(q-1) /(p-1)$ is a power of $\nu(\geq 3)$, then $n \in C_{\nu, p}^{!} \cap C_{\nu, q}^{!}$if $n \in C_{\nu, p}^{!}$and $\sigma_{p}(n)=\sigma_{q}(n)$ (and for $n \geq q^{2}$, only if). 
Moreover, if we call the primes of the form $1+(\nu-1) \nu^{k} \nu$-Fermat primes, then for $n \geq 2 \nu, p=\nu$ and a $\nu$-Fermat prime $q$ (cf. Proposition 5(ii)) we have: $n \in C_{\nu, \nu}^{!} \cap C_{\nu, q}^{!}$if and only if $n \in C_{\nu, \nu}^{!}$and $\sigma_{\nu}(n)=\sigma_{q}(n)$. Moreover, as in Proposition 1 one can prove that for $n \geq \nu^{3}-\nu^{2}+\nu$ we have: $n \in C_{\nu, \nu}^{!}$if and only if $n$ has the form $(\nu-1) \nu^{\alpha}+\nu+i, \alpha \geq 2, i=0,1, \ldots, \nu-1$. Taking into account that $\left[2 \nu, \nu^{3}-\nu^{2}+\nu\right) \cap C_{\nu, \nu}^{!}=\emptyset$, we conclude that if $n \geq 2 \nu$ and $q$ is a $\nu$-Fermat prime, then there is a bijection between $C_{\nu, \nu}^{!} \cap C_{\nu, q}^{!}$and the set of solutions of the diophantine equation

$$
\sum_{j=1}^{2 \nu-1} q^{x_{j}}=(\nu-1) \nu^{\alpha}+2 \nu-1, \quad \nu \geq 2,
$$

in integers $\alpha \geq 2,0 \leq x_{1} \leq \cdots \leq x_{2 \nu-1}$ (cf. Proposition 6).

8. Is the set $\left\{p \in P:\left|C_{p, 2}^{!} \cap C_{p, p}^{!}\right|=t\right\}$ infinite a) for $t=0$; b) for $t=2$ ?

REMARK 10. Notice that this question for $t=2$ is equivalent to the question of infinitude of primes of the form $p=e_{2}(n), n \in \mathbb{N}$. E.g., for the Mersenne primes $p=2^{k}-1, k \geq 3$, we have $e_{2}(p+1)=p$. Thus, in this case $\left|C_{p, 2}^{!} \cap C_{p, p}^{!}\right|=2$. On the other hand, for the Fermat primes $p=2^{k}+1$, $k \geq 2$, we have $e_{2}(p+1)=p-1$ and $e_{2}(p+3)=p+1+\delta_{p, 5}$. Thus, for each Fermat prime $p \geq 5$ the set $C_{p, 2}^{!} \cap C_{p, p}^{!}$is empty.

Analogously, one can show that for a prime $p$ of the form $p=2^{k}+2^{l}-1$, $2 \leq l \leq k-1$, we have $e_{2}(p+3)=p$. Thus, $\left|C_{p, 2}^{!} \cap C_{p, p}^{!}\right|=2$. On the other hand, for a prime $p$ of the form $p=2^{k}-l, 3 \leq l \leq k$, we have $e_{2}(p+l-2)<p$, $e_{2}(p+l)>p$. Therefore, $C_{p, 2}^{!} \cap C_{p, p}^{!}=\emptyset$, etc.

Acknowledgments. The author would like to express his deep gratitude to Professor Daniel Berend (Department of Mathematics and of Computer Science, Ben-Gurion University), without the fruitful conversations with whom this article most likely would not have been written. The author also wishes to thank Professor Simon Litsyn (Department of Electrical Engineering Systems, Tel-Aviv University) and Professor Andrzej Schinzel (IMPAN, Warsaw) for some important advice.

\section{References}

[1] L. J. Alex, On the diophantine equation $1+2^{a}=3^{b} 5^{c}+2^{d} 3^{e} 5^{f}$, Math. Comp. 44 (1985), 267-278.

[2] R. C. Baker, G. Harman and J. Pintz, The difference between consecutive primes, II, Proc. London Math. Soc. (3) 83 (2001), 532-562.

[3] D. Berend, Parity of exponents in the factorization of $n$ !, J. Number Theory 64 (1997), 13-19.

[4] K. Chandrasekharan, Introduction to Analytic Number Theory, Springer, Berlin, 1968. 
[5] Y.-G. Chen, On the parity of exponents in the standard factorization of $n$ !, J. Number Theory 100 (2003), 326-331.

[6] Y.-G. Chen and Y.-C. Zhu, On the prime power factorisation of n!, ibid. 82 (2000), $1-11$.

[7] P. Erdôs and R. L. Graham, Old and New Problems and Results in Combinatorial Number Theory, Enseign. Math., Univ. de Genève, Genève, 1980.

[8] R. K. Guy, Unsolved Problems in Number Theory, Springer, New York, 1981.

[9] F. Luca and P. Stanica, On the prime power factorization of $n$ !, J. Number Theory 102 (2003), 298-305.

[10] K. Prachar, Primzahlverteilung, Springer, 1957.

[11] J. C. Puchta und J. Spilker, Altes und Neues zur Quersumme, Math. Semesterber. 49 (2002), 209-226.

[12] B. Rosser, Explicit bounds for some functions of prime numbers, Amer. J. Math. 63 (1941), 211-232.

[13] J. W. Sander, On the parity of exponents in the prime factorization of factorials, J. Number Theory 90 (2001), 316-328.

[14] V. S. Shevelev, Multiplicative functions in the Fermi-Dirac arithmetic, Izv. Vyssh. Uchebn. Zaved. Sev.-Kavk. Reg. Estestv. Nauki 1996, no. 4, 28-43 (in Russian).

[15] W. Sierpiński, Elementary Theory of Numbers, North-Holland and PWN-Polish Sci. Publ., 1988.

[16] E. Trost, Primzahlen, Birkhäuser, 1953.

[17] A. Walfisz, Weylsche Exponentialsummen in der neueren Zahlentheorie, Deutsch. Verlag Wiss., Berlin, 1963.

Department of Mathematics

Ben-Gurion University of the Negev

Beer-Sheva 84105, Israel

E-mail: shevelev@bgu.ac.il 